\title{
Distributed Parameter Detection in Massive MIMO Wireless Sensor Networks Relying on Imperfect CSI
}

\author{
Apoorva Chawla, Student Member IEEE, Ajay Satyakumar Sarode, Student Member IEEE, Aditya K. \\ Jagannatham, Member IEEE and Lajos Hanzo, Fellow IEEE
}

\begin{abstract}
Distributed parameter detection is conceived for massive multiple-input multiple-output (MIMO) wireless sensor networks (WSNs), where multiple sensors collaborate to detect the presence/ absence of a spatially correlated parameter. Neyman-Pearson (NP) and generalized likelihood ratio test (GLRT)-based detectors are developed at the fusion center (FC) for known and unknown parameter detection scenarios, respectively. More explicitly, the GLRT detector also has to estimate the unknown parameter value. Closed-form expressions are derived for the probabilities of detection $\left(P_{D}\right)$ and false alarm $\left(P_{F A}\right)$ in order to characterize the performance of the proposed schemes. Furthermore, the optimal sensor transmit gains are determined for maximising the detection performance attained. An asymptotic performance analysis is carried out for determining the gain scaling laws for the massive MIMO WSN considered, when the number of antennas tends to infinity. The proposed framework is also extended to the realistic imperfect channel knowledge scenario at the FC, followed by the development of the associated fusion rules and analytical results to characterize the performance. Our simulation results closely tally the theoretical findings.
\end{abstract}

Index Terms - Wireless sensor networks (WSNs), massive multiple-input multiple-output (MIMO), generalized likelihood ratio test (GLRT), Neyman-Pearson (NP) criterion.

\section{INTRODUCTION}

WSNs, relying on miniature sensors, have attracted significant research interest in environmental and habitat monitoring, surveillance, disaster management, medical and structural monitoring, industrial applications etc. [1], [2]. Accurate detection of the parameters of interest is of pivotal importance in many WSNs to reliably establish the presence or absence of a phenomenon of interest [3]-[8]. A popular model for the implementation of a WSN is the coherent multiple access channel (MAC) based architecture, where the various sensor observations are amplified followed by their transmission to a fusion center (FC). Subsequently, using a suitably designed rule, the FC makes a decision regarding the presence or absence of the signal of interest [5]. Naturally, since the simultaneous sensor transmissions are in the same frequency

A. Chawla and A. K. Jagannatham are with the Department of Electrical Engineering, Indian Institute of Technology Kanpur, Kanpur, UP 208016 , India (e-mail: \{capoorva, adityaj\}@iitk.ac.in).

A. S. Sarode is with the Intel Corporation, Bangalore, India (e-mail: ajaysarode8794@gmail.com).

Lajos Hanzo is with the School of Electronics and Computer Science, University of Southampton, Southampton SO17 1BJ, U.K. (e-mail: lh@ecs.soton.ac.uk). This work is supported partially by the TCS Research Scholarship Program and DST-SERB Project Grant. band, the time-bandwidth resources required in such a system are independent of the number of sensors, thus making the proposed technique attractive for practical implementation in WSNs. A brief review of the related literature pertaining to parameter sensing in WSNs is presented next.

\section{A. State-of-the-Art Review}

$\mathrm{Li}$ and Dai [9] investigate the performance of a distributed detection scheme, wherein correlated sensor observations are transmitted over a MAC. As a further advance, Tepedelenlioglu and Dasarathan [10] proposed a distributed detection scheme for constant modulus sensor transmissions over a Gaussian MAC and compared its performance to other detectand-forward as well as amplify-and-forward schemes.

However, these early contributions relied on a single antenna, whilst [11], [12] used multiple antennas at the FC for improving the performance. In particular, Banavar et al. [11] employed an amplify-and-forward scheme for transmitting the sensory observations to the FC over fading multipleaccess MIMO channels. The performance of their system is characterized in terms of the error probability exponent for transmission over additive white Gaussian noise (AWGN), Rayleigh and Rician fading channels. The analysis of [11] was then further extended in [12] for determining the optimal sensor gains minimizing the error exponents, considering also different levels of CSI availability at the individual sensors. Then Nevat et al. [13] derived the optimal decision rules for a scenario wherein the sensors transmit their observations to the FC using the amplify-and-forward scheme over MIMO channels, followed by the characterization of the resultant detection and false alarm probabilities, considering also the realistic scenarios of having unknown CSI between the source and the sensors as well as the sensors and the FC. On the other hand, Ciuonzo et al. [14] designed near optimal fusion rules for MIMO WSNs, considering the transmission of local binary decisions at the sensors over a coherent MAC. Al-Jarrah et al. [15] have proposed and analyzed the performance of a novel algorithm for decision fusion in Internet of Things (IoT)-based clustered WSN. Decision rules based on local sensor decisions transmitted over an orthogonal parallel access channel (PAC) are derived for cooperative spectrum sensing in multiuser MIMO cognitive radio networks in [16]. Sensors designed for parameter detection may either employ analog or digital transmission for relaying the sensor observations 
to the FC. Due to the bandwidth and power constraints, the sensor measurements are generally compressed prior to transmission over a rate-constrained channel [17]. Typically, the local binary decisions of the individual sensors are transmitted and subsequently processed at the FC in order to arrive at a global decision. Nevertheless, this leads to performance degradation as the FC does not receive all the information [18]. Furthermore, the local detection rules have to be optimized in order to achieve the minimum detection error. As demonstrated in [19], [20], the optimal detection rule for a binary hypothesis testing problem, derived using the Neyman-Pearson (NP) and Bayesian criteria, reduces to the likelihood ratio test (LRT). However, the optimal local thresholds at the individual sensors are generally non-identical and have to be jointly evaluated along with that of the global fusion rule for reliable sensor decisions. Therefore, the search for optimal local detectors can potentially be of exponentially growing complexity [21].

Recently, several researchers have studied the benefits of employing a massive antenna array at the base station (BS) for meeting the ever-growing demand for higher data rates. In such a massive MIMO system, the BS has a very large antenna array, typically comprising hundreds of antennas, for simultaneously serving a large number of single-antenna devices/ sensors, using the same time-frequency resources [22]. This in turn leads to a high spectral efficiency in such systems. Additionally, linear receiver techniques can be adopted at the massive MIMO BS without impacting the performance [23], thereby leading to appealingly low complexity of signal processing. Furthermore, massive MIMOs facilitate a significant transmit power reduction for the devices, proportional to the number of antennas at the BS, without compromising the asymptotic rate of the users [24]-[26]. Hence, there has been a growing interest in employing massive MIMOs in WSNs [27]-[33]. In this context, Jiang et al. [27] investigated the estimation and detection performance of a WSN using a massive antenna array, wherein the sensors communicate over a coherent MAC, followed by the performance characterization of the NP and energy detectors, and the associated linear minimum mean squared error (LMMSE) estimator. Shirazinia et al. [28] investigated the problem of decentralized estimation of a correlated random parameter vector in a massive MIMO network using the MMSE estimator. The impact of transceiver hardware impairments, both at the single-antenna sensors and at the massive-antenna FC, on the detection performance of massive MIMO-based distributed detection systems, has been explored in [29]. Ciuonzo et al. [30] have proposed low complexity sub-optimal detection rules, such as the deflectionmaximizer widely linear (WL) and linear-filter fusion rules, for large antenna-array based WSNs. Ciuonzo et al. [31] have developed sub-optimal fusion rules for massive antenna based WSNs, wherein the channel is modeled by Rician fading. The authors of [32], [33] have analyzed the performance of the NP criterion based simplified fusion rules for low-SNR distributed detection in massive MIMO WSNs, incorporating also the reliabilities of the local sensor decisions. Furthermore, Dey et al. [34] propose and analyze the performance of sub-optimum decision fusion techniques conceived for an orthogonal frequency division multiplexing (OFDM) based collaborative wideband spectrum sensing system, wherein the DFC is equipped with a massive antenna array. While the benefit of massive antenna arrays is widely recognized in general communication systems, there is a paucity of contributions analyzing their benefits in the context of WSNs, especially when the sensors employ an amplify-and-forward scheme for measurement transmission, followed by parameter detection. Furthermore, none of the existing contributions have considered maximal ratio combining (MRC) at the FC in an amplify-and-forward based measurement transmission scheme for fully exploiting the benefits of a massive MIMO system, followed by the design of the optimal detectors, which can significantly reduce the complexity of parameter sensing. To fill this gap in the literature, this paper analyzes the benefit of employing a massive antenna array at the FC, wherein lowcomplexity linear processing based fusion rules are derived for detecting the presence/ absence of a spatially correlated known/ unknown parameter, employing the NP criterion and generalized likelihood ratio test (GLRT) paradigms, respectively, considering both perfect and imperfect CSI at the FC. The main contributions of this work for spatially correlated parameter detection in a massive MIMO WSN are summarized below.

\section{B. Main Contributions}

- This work considers a sensor network wherein multiple sensors transmit their amplified analog observations to the FC for the detection of a spatially correlated parameter. This is in contrast to [30]-[33], which consider the transmission of local sensor decisions to the FC. The proposed framework considers the effects of parameter correlation, which naturally exists due to the spatial proximity of the sensor nodes.

- The NP criterion and the GLRT frameworks are subsequently exploited for designing fusion rules for the detection of known and unknown spatially correlated parameters, respectively, with perfect CSI. This is different from [32], [33] that derive only the NP criterion based fusion rules using the local sensor decisions.

- To enhance the detection performance at the FC, an optimization framework is also developed for deriving the optimal sensor gains, which is absent in [13], [14], [30], [32] that are based on equal sensor power allocation.

- Closed-form analytical expressions are derived for characterizing the performance of the proposed detectors in terms of the resultant $P_{D}$ and $P_{F A}$ at the FC.

- The above schemes and analysis are also extended to practical scenarios having imperfect CSI, which includes determining the requisite detectors and characterizing their analytical detection performance. By contrast, [11], [12], [14] only consider the idealized scenario of perfect CSI at the FC.

- The asymptotic performance of the fusion rules is examined in the large antenna regime and the pertinent scaling laws are derived for both uniform and optimized gain allocation schemes in the massive MIMO WSN scenarios, whereas [30], [33] characterize the asymptotic 
TABLE I

A BRIEF COMPARISON WITH EXISTING LITERATURE

\begin{tabular}{|c|c|c|c|c|c|c|c|c|c|c|c|c|}
\hline & [11] & [12] & [13] & {$[14]$} & [27] & [28] & [29] & {$[30]$} & [31] & [32] & {$[33]$} & Proposed Work \\
\hline Massive MIMO Architecture & & & & & $\overline{\bar{\checkmark}}$ & $\overline{\bar{\checkmark}}$ & $\overline{\bar{\checkmark}}$ & $\overline{\bar{\checkmark}}$ & $\overline{\bar{\checkmark}}$ & $\bar{\nabla} \checkmark$ & $\overline{\bar{\checkmark}}$ & $\overline{\mathrm{V}}$ \\
\hline Local Sensor Decisions & & & & $\bar{\checkmark}$ & & & & $\checkmark$ & $\checkmark$ & $\checkmark$ & $\checkmark$ & \\
\hline Amplify \& Forward Scheme & $\checkmark$ & $\checkmark$ & $\checkmark$ & & $\checkmark$ & $\checkmark$ & $\checkmark$ & & & & & $\checkmark$ \\
\hline Optimal Gain/ Power Allocation & & $\bar{\checkmark}$ & & & $\bar{\checkmark}$ & $\bar{\checkmark}$ & $\bar{\checkmark}$ & & & & & $\bar{\checkmark}$ \\
\hline Single-Antenna Sensors/Users & $\sqrt{ }$ & $\checkmark$ & $\checkmark$ & $\checkmark$ & $\checkmark$ & $\checkmark$ & $\checkmark$ & $\sqrt{ }$ & $\sqrt{ }$ & $\sqrt{ }$ & $\checkmark$ & $\checkmark$ \\
\hline Signaling Matrix Optimization & & & & & & & & & & & $\bar{\checkmark}$ & \\
\hline Gain/ Power Scaling & & & & & $\sqrt{ }$ & $\sqrt{ }$ & $\sqrt{ }$ & $\bar{\checkmark}$ & & & $\sqrt{ }$ & $\checkmark$ \\
\hline Spatially Correlated Parameter & & & & & & $\checkmark$ & & & & & & $\checkmark$ \\
\hline Linear Filtering Architecture at FC & & & & & & & & $\sqrt{ }$ & & $\checkmark$ & $\sqrt{ }$ & $\checkmark$ \\
\hline Multiple Observation Vector Model & & & $\checkmark$ & & & & & & & $\checkmark$ & $\bar{\checkmark}$ & \\
\hline Low SNR Approximation & & & & & & & & & & $\sqrt{ }$ & $\sqrt{ }$ & \\
\hline
\end{tabular}

performance only for uniform power allocation. It is explicitly demonstrated that the transmit gain of each sensor can be reduced in proportion to the number of antennas at the $\mathrm{FC}$, which is key to maximizing the lifetime of the battery operated sensor nodes.

\section{Organization}

The rest of the paper is organized as follows. Section II describes the system model for spatially correlated parameter sensing and distributed detection in massive MIMO WSNs, where the sensors transmit their amplified observations to the FC over a MAC. Section III presents the log likelihood ratio (LLR) based fusion rule for the detection of a known parameter, followed by its detection performance characterization. Subsequently we also develop the optimization framework of deriving the optimal sensor gains. This framework is then extended to an unknown parameter detection scenario in Section IV, wherein a GLRT-based detector is derived. Our asymptotic performance analysis and our gain scaling laws are derived in Section $\mathrm{V}$ for the massive MIMO detectors proposed in Section III and Section IV. Our imperfect CSI framework is presented in Section VI, followed by the fusion rules conceived both for known and unknown parameter detection in Section VI-A and Section VI-B, respectively. Our simulation results are provided in Section VII, followed by our conclusions in Section VIII.

\section{Notations}

The notation used throughout the paper is summarized as follows. Uppercase boldface $(\mathbf{X})$ and lowercase boldface $(\mathbf{x})$ represent matrices and vectors, respectively, while $[\mathbf{X}]_{m n}$ and $x_{n}$ denote the $(m, n)$ th and $n$th element of the matrix $\mathbf{X}$ and vector $\mathbf{x}$, respectively. Furthermore, $\mathbf{0}$ denotes a vector with all its elements being zero and $\mathbf{I}$ represents an identity matrix. The operators $\mathbb{E}\{\cdot\}, \Re\{\cdot\},(\cdot)^{T},(\cdot)^{H}, \exp \{\cdot\},(\cdot)^{-1}$, $|\cdot|$ and $\|\cdot\|_{\mathbf{A}}$ represent the expectation, real part, transpose, Hermitian, exponential, inverse, absolute value and weighted norm, respectively and the symbol $\stackrel{a}{\sim}$ is used for defining an approximate distribution of a random variable. The quantity $p(\cdot)$ represents the probability density function (PDF) and $p(A ; B)$ represents the PDF where $A$ is parameterized by $B$. The notation $\mathbf{x} \sim \mathcal{C N}(\boldsymbol{\mu}, \mathbf{C})$ is used to define the complex Gaussian distribution of the random vector $\mathbf{x}$ with mean $\boldsymbol{\mu}$ and covariance matrix $\mathbf{C}$. The term $Q$ represents the Gaussian $Q$-function and is defined as $Q(x)=\frac{1}{\sqrt{2 \pi}} \int_{x}^{\infty} \exp \left(-\frac{y^{2}}{2}\right) d y$.

\section{System MODEL}

Let us consider a WSN wherein $K$ sensors monitor a signal of interest belonging to the set $\Theta \in\{0, \theta\} \in \mathbb{R}$ for differentiating between the events characterized by the set of hypotheses $\mathcal{H}=\left\{\mathcal{H}_{0}, \mathcal{H}_{1}\right\}$. The null hypothesis $\mathcal{H}_{0}$ corresponding to $\Theta=0$, represents the absence of the signal of interest and the alternative hypothesis $\mathcal{H}_{1}$ denotes the presence of the signal, i.e., $\Theta=\theta$. Each sensor node has a single antenna and communicates with a FC equipped with a large antenna array comprising of $M$ antennas, where we have $M \gg K$. Note that most existing contributions on massive MIMO [24], [26], [30], [33] consider single antenna devices and sensors to limit the device complexity. The sensors transmit their suitably preprocessed correlated measurements to the FC over a coherent MAC, as illustrated in Fig. 1. The measurement process at the $j$-th sensor, $1 \leq j \leq K$, can be modeled as $x_{j}=a_{j} \Theta+v_{j}$, where $v_{j}$ is the complex Gaussian observation noise at the $j$-th sensor that is distributed as $v_{j} \sim \mathcal{C N}\left(0, \sigma_{v}^{2}\right)$ and $a_{j}$ are observation constants, similar to the models in [35]. The $j$-th sensor measurement $x_{j}$ is thereafter precoded using a complex gain $f_{j}$ and forwarded to the FC for a global decision. The signal $\mathbf{y} \in \mathbb{C}^{M \times 1}$ received by the FC under the two hypotheses can be expressed as

$$
\begin{aligned}
& \mathcal{H}_{0}: \mathbf{y}=\mathbf{G F v}+\mathbf{n} \\
& \mathcal{H}_{1}: \mathbf{y}=\mathbf{G F a} \theta+\mathbf{G F v}+\mathbf{n},
\end{aligned}
$$

where $\mathbf{n} \in \mathbb{C}^{M \times 1}$ is the AWGN vector at the FC that is distributed as $\mathbf{n} \sim \mathcal{C N}\left(\mathbf{0}, \sigma_{n}^{2} \mathbf{I}_{M}\right), \mathbf{a}=\left[a_{1}, \ldots, a_{K}\right]^{T} \in \mathbb{C}^{K \times 1}$ and $\mathbf{v} \in \mathbb{C}^{K \times 1}$ denotes a similar stacking of the observation noise samples $v_{j}$ of the $K$ sensors. The diagonal matrix $\mathbf{F}=\operatorname{diag}\left(f_{1}, \ldots, f_{K}\right) \in \mathbb{C}^{K \times K}$ is obtained by placing the complex gains $f_{j}, 1 \leq j \leq K$, along its principal diagonal. The channel matrix between the $K$ sensors and the FC is represented by $\mathbf{G}=\left[\mathbf{g}_{1}, \ldots, \mathbf{g}_{K}\right] \in \mathbb{C}^{M \times K}$ and its $j$-th column vector, which corresponds to the $j$-th sensor, is represented by $\mathbf{g}_{j} \in \mathbb{C}^{M \times 1}$. The $m$-th, $1 \leq m \leq M$, element of $\mathbf{g}_{j}$, denoted by $g_{m j}=[\mathbf{G}]_{m j}$ can be modeled as $g_{m j}=\sqrt{\beta_{j}} h_{m j}$, where $h_{m j}$ denotes the small-scale fading coefficient between the $m$ th antenna at the FC and the $j$-th sensor. The large-scale fading coefficient $\beta_{j}$ that models the geometric attenuation and lognormal shadowing is invariant over $m$, and it is assumed to be constant over multiple coherence time intervals. Therefore, the channel matrix $\mathbf{G}$ can be decomposed as

$$
\mathbf{G}=\mathbf{H D}^{1 / 2}
$$




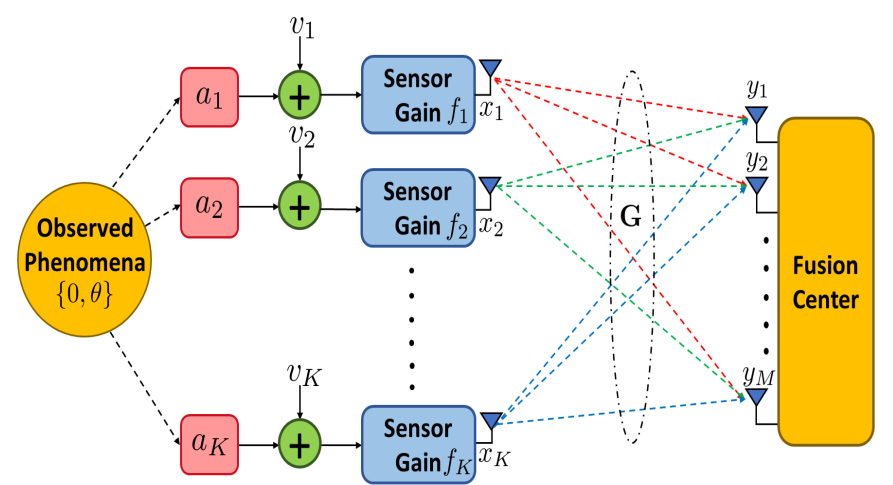

Fig. 1. System model of the massive MIMO WSN, wherein $K$ single-antenna sensors communicate with a $M$ receive antenna array based FC.

where $\mathbf{H}=\left[\mathbf{h}_{1}, \ldots, \mathbf{h}_{K}\right]$ and $\mathbf{D}=\operatorname{diag}\left(\beta_{1}, \ldots, \beta_{K}\right)$. The diagonal matrix $\mathbf{D} \in \mathbb{C}^{K \times K}$ represents the large-scale fading matrix and $\mathbf{H} \in \mathbb{C}^{M \times K}$ denotes the small-scale fading matrix comprising the fast fading channel coefficients between the $K$ sensors and the FC. The elements of $\mathbf{H}$, denoted by $h_{m j}$, are assumed to be independent identically distributed (i.i.d.) as $\mathcal{C N}(0,1)$. Consequently, under favorable propagation conditions, the elements of $\mathbf{G}$ can also be modeled as independent random variables that are distributed as $g_{m j} \sim \mathcal{C N}\left(0, \beta_{j}\right)$. Moreover, the channel vectors corresponding to distinct sensors are assumed to be mutually independent. This holds true as a result of the assumption that the sensors are spatially well separated in a typical WSN. Therefore, from the law of large numbers, this leads to the property

$$
\frac{1}{M} \mathbf{G}^{H} \mathbf{G}=\mathbf{D}^{1 / 2} \frac{\mathbf{H}^{H} \mathbf{H}}{M} \mathbf{D}^{1 / 2} \approx \mathbf{D}, \text { for } M \gg K .
$$

Hence, as $\mathrm{M}$ becomes large, under favorable propagation conditions, the channel vectors of different sensors become pairwise orthogonal. This is a widely exploited property of massive MIMO systems, which has been exploited in numerous contributions [24], [36]. For the above system, the optimal decision rules and the resultant performance at the FC are determined for various scenarios.

\section{Fusion Rule For a KNOWn Signal $\theta$ With PERFECT CSI}

This section derives the fusion rule for the detection of a known deterministic parameter $\theta$ with perfect CSI. The more challenging scenarios with unknown $\theta$ and imperfect CSI are considered later in Section IV and Section VI, respectively. Using the popular NP criterion [37], which aims for maximizing the probability of detection subject to a given probability of false alarm, the LLR test formulated for the binary hypothesis testing problem in (1) can be expressed as

$$
T(\mathbf{y})=\ln \left[\frac{p\left(\mathbf{y} \mid \mathcal{H}_{1}\right)}{p\left(\mathbf{y} \mid \mathcal{H}_{0}\right)}\right] \underset{\mathcal{H}_{0}}{\stackrel{\mathcal{H}_{1}}{\gtrless}} \gamma,
$$

where $p\left(\mathbf{y} \mid \mathcal{H}_{0}\right)$ and $p\left(\mathbf{y} \mid \mathcal{H}_{1}\right)$ denote the likelihood functions corresponding to the null and alternative hypotheses, respectively, while $\gamma$ represents the detection threshold. It can be observed that the received signal in (1) follows the Gaussian

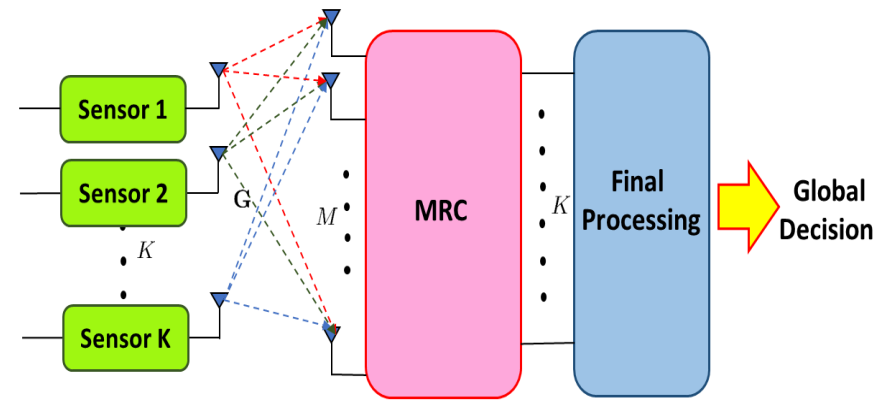

Fig. 2. Two-step architecture at the FC.

distribution under both the hypotheses, which is given as

$$
\mathbf{y} \sim\left\{\begin{array}{cc}
\mathcal{C N}(\mathbf{0}, \tilde{\mathbf{C}}) & , \text { under } \mathcal{H}_{0} \\
\mathcal{C N}(\mathbf{G F a} \theta, \tilde{\mathbf{C}}) & , \text { under } \mathcal{H}_{1}
\end{array}\right.
$$

where $\tilde{\mathbf{C}}=\sigma_{v}^{2} \mathbf{G F F}{ }^{H} \mathbf{G}^{H}+\sigma_{n}^{2} \mathbf{I}$ denotes the covariance of the effective noise at the output. Substituting the PDFs of the observation $\mathbf{y}$, the LLR test in (4) can be expressed as

$$
T(\mathbf{y})=\ln \left[\frac{\exp \left(-(\mathbf{y}-\mathbf{G F a} \theta)^{H} \tilde{\mathbf{C}}^{-1}(\mathbf{y}-\mathbf{G F a} \theta)\right)}{\exp \left(-\mathbf{y}^{H} \tilde{\mathbf{C}}^{-1} \mathbf{y}\right)}\right] .
$$

The FC can directly employ the LLR test in (5) for the detection of the signal of interest. However, the above test is computationally complex as it requires the matrix inversion $\tilde{\mathbf{C}}^{-1} \in \mathbb{C}^{M \times M}$, which imposes a computational complexity of $\mathcal{O}\left(M^{2} K\right)$ that depends on $M$. Interestingly, it has been widely exploited in the existing literature on massive MIMO systems that linear processing employing MRC achieves good performance in the large antenna regime, i.e., when $M \gg K$ [26]. Exploiting this property, a two-step procedure is developed now for efficiently computing the fusion rules with reduced computational complexities, which are suitable for practical implementation. Fig. 2 shows a block diagram of the system. In the first step, the received signal is processed using the MRC at the FC. Subsequently, the processed measurements of the individual sensors are combined in the second step to obtain a global decision.

Upon applying the MRC to the received signal in (1), the filter output $\tilde{\mathbf{y}} \in \mathbb{C}^{K \times 1}$ corresponding to the hypotheses $\mathcal{H}_{0}$ and $\mathcal{H}_{1}$, respectively, can be expressed as

$$
\begin{aligned}
& \mathcal{H}_{0}: \tilde{\mathbf{y}}=\frac{1}{M} \mathbf{G}^{H} \mathbf{y}=\frac{\mathbf{G}^{H} \mathbf{G}}{M} \mathbf{F} \mathbf{v}+\frac{1}{M} \mathbf{G}^{H} \mathbf{n} \\
& \mathcal{H}_{1}: \tilde{\mathbf{y}}=\frac{1}{M} \mathbf{G}^{H} \mathbf{y}=\frac{\mathbf{G}^{H} \mathbf{G}}{M} \mathbf{F a} \theta+\frac{\mathbf{G}^{H} \mathbf{G}}{M} \mathbf{F} \mathbf{v}+\frac{1}{M} \mathbf{G}^{H} \mathbf{n} .
\end{aligned}
$$

Under the favorable propagation conditions described in (3), the filter output in (6) can be closely approximated for a massive MIMO scenario as

$$
\begin{aligned}
& \mathcal{H}_{0}: \tilde{\mathbf{y}} \approx \tilde{\mathbf{n}} \\
& \mathcal{H}_{1}: \tilde{\mathbf{y}} \approx \mathbf{D F a} \theta+\tilde{\mathbf{n}},
\end{aligned}
$$

where the noise vector $\tilde{\mathbf{n}}=\mathbf{D F} \mathbf{v}+\frac{1}{M} \mathbf{G}^{H} \mathbf{n} \in \mathbb{C}^{K \times 1}$ is distributed as $\mathcal{C N}(\mathbf{0}, \mathbf{C})$ with $\mathbf{C}=\sigma_{v}^{2} \mathbf{D F F}{ }^{H} \mathbf{D}^{H}+\frac{\sigma_{n}^{2}}{M} \mathbf{D}$. The distribution of $\tilde{\mathbf{y}}$ under both the hypotheses can be equivalently written as

$$
\begin{aligned}
& \mathcal{H}_{0}: \tilde{\mathbf{y}} \stackrel{a}{\sim} \mathcal{C N}(\mathbf{0}, \mathbf{C}) \\
& \mathcal{H}_{1}: \tilde{\mathbf{y}} \stackrel{a}{\sim} \mathcal{C N}(\mathbf{D F a} \theta, \mathbf{C}) .
\end{aligned}
$$


TABLE II

COMPUTATIONAL COMPLEXITY OF FUSION RULES

\begin{tabular}{|c|c|}
\hline Fusion Rules & Complexity \\
\hline \hline$T_{\mathrm{KP}}(\mathbf{y})$ & $\mathcal{O}\left(M^{2} K\right)$ \\
\hline$T_{\mathrm{KP}}(\tilde{\mathbf{y}})$ & $\mathcal{O}\left(M K+K^{3}\right)$ \\
\hline$T_{\mathrm{UP}}(\mathbf{y})$ & $\mathcal{O}\left(M^{2} K\right)$ \\
\hline$T_{\mathrm{UP}}(\tilde{\mathbf{y}})$ & $\mathcal{O}\left(M K+K^{3}\right)$ \\
\hline$T_{\mathrm{KIP}}(\mathbf{y})$ & $\mathcal{O}\left(M^{2} K+M \tau K\right)$ \\
\hline$T_{\mathrm{KIP}}(\breve{\mathbf{y}})$ & $\mathcal{O}\left(M \tau K+K^{3}\right)$ \\
\hline$T_{\mathrm{UIP}}(\mathbf{y})$ & $\mathcal{O}\left(M^{2} K+M \tau K\right)$ \\
\hline$T_{\mathrm{UIP}}(\breve{\mathbf{y}})$ & $\mathcal{O}\left(M \tau K+K^{3}\right)$ \\
\hline
\end{tabular}

The LLR for the binary hypothesis testing problem in (7) can now be expressed as

$$
T_{\mathrm{KP}}(\tilde{\mathbf{y}})=\ln \left[\frac{p\left(\tilde{\mathbf{y}} \mid \mathcal{H}_{1}\right)}{p\left(\tilde{\mathbf{y}} \mid \mathcal{H}_{0}\right)}\right]
$$

which can be further simplified using the PDFs determined in (8) under both hypotheses as

$$
\begin{aligned}
T_{\mathrm{KP}}(\tilde{\mathbf{y}}) & \equiv \ln \left[\frac{\exp \left(-(\tilde{\mathbf{y}}-\mathbf{D F} \mathbf{a} \theta)^{H} \mathbf{C}^{-1}(\tilde{\mathbf{y}}-\mathbf{D F a} \theta)\right)}{\exp \left(-\left(\tilde{\mathbf{y}}^{H} \mathbf{C}^{-1} \tilde{\mathbf{y}}\right)\right)}\right] \\
& \equiv \Re\left(\mathbf{a}^{H} \mathbf{F}^{H} \mathbf{D}^{H} \mathbf{C}^{-1} \tilde{\mathbf{y}}\right) .
\end{aligned}
$$

An alternative derivation of the test statistic using an asymptotic simplification of the LRT is given in the technical report in [38]. It is worth noting that the above test, in contrast to the LLR test in (5), is numerically stable and has a low computational complexity because of the diagonal structure of the covariance matrix $\mathbf{C} \in \mathbb{C}^{K \times K}$. The computational complexity of evaluating $\mathbf{C}^{-1}$ is $\mathcal{O}\left(K^{3}\right)$, which does not depend on $M$, in contrast to the complexity required to evaluate $\tilde{\mathbf{C}}^{-1}$ as mentioned above. Furthermore, the computational complexity of the proposed simplified detector $T_{\mathrm{KP}}(\tilde{\mathbf{y}})$ in (10), based on the filtered output $\tilde{\mathbf{y}}$, is $\mathcal{O}\left(M K+K^{3}\right)$, while the original test $T(\mathbf{y})$ in (5) has a computational complexity of $O\left(M^{2} K\right)$. Table II summarizes the computational complexities of the various fusion rules. Upon substituting the matrices $\mathbf{F}, \mathbf{D}$, $\mathbf{C}$ and the vectors $\tilde{\mathbf{y}}$, a in the above expression (10), the closed-form expression for the simplified test statistic can be expressed as

$$
T_{\mathrm{KP}}(\tilde{\mathbf{y}}) \equiv \sum_{j=1}^{K} \Re\left(\frac{M a_{j}^{*} f_{j}^{*} \tilde{y}_{j}}{\sigma_{v}^{2} M \beta_{j}\left|f_{j}\right|^{2}+\sigma_{n}^{2}}\right) \underset{\mathcal{H}_{0}}{\stackrel{\mathcal{H}_{1}}{\gtrless}} \gamma^{\prime},
$$

where $\gamma^{\prime}$ denotes the detection threshold. As seen, the above detector is a simple linear filter, which is well suited for practical implementation due to its low complexity. For the specific scenario where all the sensor transmit gains are assumed to be same, i.e., $\left|f_{j}\right|^{2}=p_{u}, \forall j$, the above test statistic further reduces to $T_{\mathrm{KP}, \mathrm{I}}(\tilde{\mathbf{y}}) \equiv \sum_{j=1}^{K} \Re\left(\frac{\sqrt{p_{u}} M a_{j}^{*} \tilde{y}_{j}}{\sigma_{v}^{2} p_{u} M \beta_{j}+\sigma_{n}^{2}}\right)$. The probabilities of detection $\left(P_{D}\right)$ and false alarm $\left(P_{F A}\right)$ of this test $T_{\mathrm{KP}}(\tilde{\mathbf{y}})$ are given by the result below.

Theorem 1. For a given threshold $\gamma^{\prime}$, the probabilities of detection and false alarm of the test statistic $T_{K P}(\tilde{\mathbf{y}})$ in (11), denoted by $P_{D}$ and $P_{F A}$, respectively, can be formulated as

$$
P_{D}=Q\left(\frac{\gamma^{\prime}-\mu_{T_{K P} \mid \mathcal{H}_{1}}}{\sigma_{T_{K P}}}\right), P_{F A}=Q\left(\frac{\gamma^{\prime}-\mu_{T_{K P} \mid \mathcal{H}_{0}}}{\sigma_{T_{K P}}}\right),
$$

where the terms $\mu_{T_{K P} \mid \mathcal{H}_{0}}, \mu_{T_{K P} \mid \mathcal{H}_{1}}$ are the means under the null and alternative hypotheses, respectively, and $\sigma_{T_{K P}}^{2}$ denotes the variance of $T_{K P}(\tilde{\mathbf{y}})$, yielding:

$$
\begin{aligned}
\mu_{T_{K P} \mid \mathcal{H}_{0}} & =0 \\
\mu_{T_{K P} \mid \mathcal{H}_{1}} & =\sum_{j=1}^{K} \frac{M \beta_{j}\left|a_{j}\right|^{2}\left|f_{j}\right|^{2} \theta}{\sigma_{v}^{2} M \beta_{j}\left|f_{j}\right|^{2}+\sigma_{n}^{2}} \\
\sigma_{T_{K P}}^{2} & =\sum_{j=1}^{K} \frac{1}{2}\left(\frac{M \beta_{j}\left|a_{j}\right|^{2}\left|f_{j}\right|^{2}}{\sigma_{v}^{2} M \beta_{j}\left|f_{j}\right|^{2}+\sigma_{n}^{2}}\right),
\end{aligned}
$$

Proof: Given in Appendix A.

\section{A. Sensor Gain Optimization}

This subsection formulates our optimization framework for determining the optimal sensor gains $\left|f_{j}\right|^{2}, 1 \leq j \leq K$, similar to the approach in [27]. Our goal is that of further enhancing the detection performance of the test proposed in (11) via the optimal sharing of the total sensor power $P$. The deflection coefficient ${ }^{1}$ defined in [39] for characterizing the detection performance of the test in (11) can be expressed as

$$
d^{2}=\frac{\left(\mu_{T_{\mathrm{KP}} \mid \mathcal{H}_{1}}-\mu_{T_{\mathrm{KP}} \mid \mathcal{H}_{0}}\right)^{2}}{\sigma_{T_{\mathrm{KP}}}^{2}} .
$$

Upon substituting the expressions of $\mu_{T_{\mathrm{KP}} \mid \mathcal{H}_{0}}, \mu_{T_{\mathrm{KP}} \mid \mathcal{H}_{1}}$ and $\sigma_{T_{\mathrm{KP}}}^{2}$ from (13), (14) and (15), respectively, into (16), the deflection coefficient expression above can be further simplified to

$$
d_{\mathrm{KP}}^{2}=\sum_{j=1}^{K} \frac{2 M \theta^{2} \beta_{j}\left|a_{j}\right|^{2}\left|f_{j}\right|^{2}}{\sigma_{v}^{2} M \beta_{j}\left|f_{j}\right|^{2}+\sigma_{n}^{2}}=2 \theta \mu_{T_{\mathrm{KP}} \mid \mathcal{H}_{1}} .
$$

Thus, maximizing $\mu_{T_{\mathrm{KP}} \mid \mathcal{H}_{1}}$ will also maximize the deflection coefficient, which in turn maximizes the detection performance. Defining $p_{j}=\left|f_{j}\right|^{2}$, the optimization problem constructed for maximizing the deflection coefficient can be formulated as

$$
\begin{gathered}
\min _{p_{j}} \sum_{j=1}^{K} \frac{-M \beta_{j}\left|a_{j}\right|^{2} p_{j}}{\sigma_{v}^{2} M \beta_{j} p_{j}+\sigma_{n}^{2}} \\
\text { subject to } \sum_{j=1}^{K} p_{j}=P \\
p_{j} \geq 0,
\end{gathered}
$$

where $P$ denotes the total sensor transmit power. The result below determines the optimal sensor gains $p_{j}$.

Lemma 1. The optimal values of $p_{j}$ that are the solution of the optimization problem in (18) are given as

$$
p_{j}=\left(\sqrt{\frac{\sigma_{n}^{2}\left|a_{j}\right|^{2}}{\lambda M \beta_{j} \sigma_{v}^{4}}}-\frac{\sigma_{n}^{2}}{M \beta_{j} \sigma_{v}^{2}}\right)^{+},
$$

where $(x)^{+}=x$ for $x \geq 0$ and 0 otherwise. The dual variable $\lambda>0$ is evaluated using the constraint $\sum_{j=1}^{K} p_{j}=P$.

Proof: See Appendix B.

${ }^{1}$ It is a normalized squared distance between the centroids of the PDFs of $T_{\mathrm{KP}}(\tilde{\mathbf{y}})$ under both hypotheses, with the normalization factor being the variance. 
The lower and upper bounds on $\lambda$ denoted by $\lambda_{l}, \lambda_{u}$, respectively, can be represented as $\lambda_{l}=\min _{j}\left\{\frac{\sigma_{n}^{2} M \beta_{j}\left|a_{j}\right|^{2}}{\left(\sigma_{v}^{2} P M \beta_{j}+\sigma_{n}^{2}\right)^{2}}\right\}$ and $\lambda_{u}=\max _{j} .\left\{\frac{M \beta_{j}\left|a_{j}\right|^{2}}{\sigma_{n}^{2}}\right\}$. The value of $\lambda$ is obtained by the classic bisection search over $\left[\lambda_{l}, \lambda_{u}\right]$. The next section considers a scenario where the signal is unknown. The pertinent fusion rule and the corresponding performance analysis based on the aforementioned framework for massive MIMO WSNs are discussed next.

\section{Fusion Rule for UnKNOWN $\theta$ With PERfect CSI}

In this section, a GLRT based hypothesis testing framework [37] is presented for the detection of an unknown signal. Let $\hat{\theta}$ denote the maximum likelihood (ML) estimate of the parameter $\theta$ under the alternative hypothesis $\mathcal{H}_{1}$ that is obtained by maximizing the pdf of the MRC output $\tilde{\mathbf{y}}$ under the alternative hypothesis, i.e., $p\left(\tilde{\mathbf{y}} ; \theta \mid \mathcal{H}_{1}\right)$, which is given as [40]

$p\left(\tilde{\mathbf{y}} ; \theta \mid \mathcal{H}_{1}\right)=\frac{1}{\pi^{K}|\mathbf{C}|} \exp \left[-(\tilde{\mathbf{y}}-\mathbf{D F a} \theta){ }^{H} \mathbf{C}^{-1}(\tilde{\mathbf{y}}-\mathbf{D F a} \theta)\right]$.

The value of $\hat{\theta}$ is determined as

$$
\hat{\theta}=\frac{\Re\left(\mathbf{a}^{H} \mathbf{F}^{H} \mathbf{D}^{H} \mathbf{C}^{-1} \tilde{\mathbf{y}}\right)}{\mathbf{a}^{H} \mathbf{F}^{H} \mathbf{D}^{H} \mathbf{C}^{-1} \mathbf{D F} \mathbf{a}}
$$

The PDFs of the filter output vector $\tilde{\mathbf{y}}$ under both the hypotheses can be obtained as

$$
\begin{aligned}
p\left(\tilde{\mathbf{y}} ; \hat{\theta} \mid \mathcal{H}_{1}\right) & =\frac{1}{\pi^{K}|\mathbf{C}|} \exp \left(-\|\tilde{\mathbf{y}}-\mathbf{D F a} \hat{\theta}\|_{\mathbf{C}^{-1}}^{2}\right) \\
p\left(\tilde{\mathbf{y}} \mid \mathcal{H}_{0}\right) & =\frac{1}{\pi^{K}|\mathbf{C}|} \exp \left(-\tilde{\mathbf{y}}^{H} \mathbf{C}^{-1} \tilde{\mathbf{y}}\right) .
\end{aligned}
$$

Using (21), the GLRT statistic $T_{\mathrm{UP}}(\tilde{\mathbf{y}})$ for the massive MIMO WSN can be simplified to

$$
\begin{aligned}
T_{\mathrm{UP}}(\tilde{\mathbf{y}}) & =\ln \left[\frac{p\left(\tilde{\mathbf{y}} ; \hat{\theta} \mid \mathcal{H}_{1}\right)}{p\left(\tilde{\mathbf{y}} \mid \mathcal{H}_{0}\right)}\right] \\
& \equiv \ln \left[\frac{\frac{1}{\pi^{K}|\mathbf{C}|} \exp \left(-(\tilde{\mathbf{y}}-\mathbf{D F a} \hat{\theta})^{H} \mathbf{C}^{-1}(\tilde{\mathbf{y}}-\mathbf{D F a} \hat{\theta})\right)}{\frac{1}{\pi^{K}|\mathbf{C}|} \exp \left(-\tilde{\mathbf{y}}^{H} \mathbf{C}^{-1} \tilde{\mathbf{y}}\right)}\right] \\
& \equiv \Re\left(\hat{\theta} \mathbf{a}^{H} \mathbf{F}^{H} \mathbf{D}^{H} \mathbf{C}^{-1} \tilde{\mathbf{y}}\right) .
\end{aligned}
$$

Upon substituting the value of $\hat{\theta}$ obtained in (20) into (22), the test statistic can be further simplified to obtain the test

$$
T_{\mathrm{UP}}(\tilde{\mathbf{y}}) \equiv\left|\sum_{j=1}^{K} \Re\left(\frac{M f_{j}^{*} a_{j}^{*} \tilde{y}_{j}}{\sigma_{v}^{2} M \beta_{j}\left|f_{j}\right|^{2}+\sigma_{n}^{2}}\right)\right| \equiv\left|T_{\mathrm{UP}}^{\prime}(\tilde{\mathbf{y}})\right| \underset{\mathcal{H}_{0}}{\stackrel{\mathcal{H}_{1}}{\gtrless}} \gamma^{\prime \prime} .
$$

The detection performance of the test above is given by the result below.

Theorem 2. The $P_{D}$ and $P_{F A}$ corresponding to the test statistic $T_{U P}(\tilde{\mathbf{y}})$ in (23) for the detection of an unknown deterministic parameter in a massive MIMO WSN are given as

$$
\begin{aligned}
P_{D} & =Q\left(\frac{\gamma^{\prime \prime}-\mu_{T_{U P}^{\prime} \mid \mathcal{H}_{1}}}{\sigma_{T_{U P}^{\prime}}}\right)+Q\left(\frac{\gamma^{\prime \prime}+\mu_{T_{U P}^{\prime} \mid \mathcal{H}_{1}}}{\sigma_{T_{U P}^{\prime}}}\right), \\
P_{F A} & =2 Q\left(\frac{\gamma^{\prime \prime}}{\sigma_{T_{U P}^{\prime}}^{\prime}}\right),
\end{aligned}
$$

where $\gamma^{\prime \prime}$ represents the detection threshold. The quantities $\mu_{T_{U P}^{\prime} \mid \mathcal{H}_{0}}, \mu_{T_{U P}^{\prime} \mid \mathcal{H}_{1}}$ denote the means under the pair of hypotheses $\mathcal{H}_{0}$ and $\mathcal{H}_{1}$, respectively and $\sigma_{T_{U P}^{\prime}}^{2}$ is the variance under both the hypotheses, which are given as

$$
\begin{aligned}
\mu_{T_{U P}^{\prime} \mid \mathcal{H}_{0}} & =0 \\
\mu_{T_{U P}^{\prime} \mid \mathcal{H}_{1}} & =\sum_{j=1}^{K} \frac{M \beta_{j}\left|a_{j}\right|^{2}\left|f_{j}\right|^{2} \theta}{\sigma_{v}^{2} M \beta_{j}\left|f_{j}\right|^{2}+\sigma_{n}^{2}}, \\
\sigma_{T_{U P}^{\prime}}^{2} & =\sum_{j=1}^{K} \frac{1}{2}\left(\frac{M \beta_{j}\left|a_{j}\right|^{2}\left|f_{j}\right|^{2}}{\sigma_{v}^{2} M \beta_{j}\left|f_{j}\right|^{2}+\sigma_{n}^{2}}\right) .
\end{aligned}
$$

Proof: See Appendix C.

\section{A. Sensor Gain Optimization}

Similar to the known parameter case, the detection performance for the unknown parameter scenario can be maximized by maximizing the deflection coefficient of the test $T_{\mathrm{UP}}(\tilde{\mathbf{y}})$ in (23). Using (24), the detection threshold $\gamma^{\prime \prime}$ can be represented in terms of the $P_{F A}$ as $\gamma^{\prime \prime}=\left[Q^{-1}\left(P_{F A} / 2\right)\right] \sigma_{T_{\mathrm{UP}}^{\prime}}$. Upon substituting $\gamma^{\prime \prime}$ in (24), the probability of detection $P_{D}$ for the test can be recast as $P_{D}=Q\left(Q^{-1}\left(P_{F A} / 2\right)-\sqrt{d_{\mathrm{UP}}^{2}}\right)+$ $Q\left(Q^{-1}\left(P_{F A} / 2\right)+\sqrt{d_{\mathrm{UP}}^{2}}\right)$, where $d_{\mathrm{UP}}^{2}$ denotes the deflection coefficient corresponding to the test statistic $T_{\mathrm{UP}}(\tilde{\mathbf{y}})$ in (23) that can be expressed as

$$
d_{\mathrm{UP}}^{2}=\frac{\left(\mu_{T_{\mathrm{UP}}^{\prime} \mid \mathcal{H}_{1}}\right)^{2}}{\sigma_{T_{\mathrm{UP}}^{\prime}}^{2}}=2 \theta \mu_{T_{\mathrm{UP}}^{\prime} \mid \mathcal{H}_{1}} .
$$

Using (28), the optimization problem determining the optimal values $p_{j}=\left|f_{j}\right|^{2}$ for improving the detection performance can be formulated as

$$
\begin{gathered}
\min _{p_{j}} \sum_{j=1}^{K} \frac{-M \beta_{j}\left|a_{j}\right|^{2} p_{j}}{\sigma_{v}^{2} M \beta_{j} p_{j}+\sigma_{n}^{2}} \\
\text { subject to } \sum_{j=1}^{K} p_{j}=P \\
p_{j} \geq 0 .
\end{gathered}
$$

As seen, the above optimization problem is similar to that for the known parameter scenario of (18) and the solution is therefore once again given by (19). The asymptotic performance analysis and the pertinent gain scaling laws for the massive MIMO WSN for various scenarios are determined in the subsequent section.

\section{Asymptotic Performance Analysis}

This section analyzes the asymptotic performance of the proposed detectors for the deterministic known and unknown parameter scenarios of (11) and (23), respectively, in the large antenna regime, i.e. when $M \rightarrow \infty$, considering both identical and optimized sensor gains. The asymptotic performance corresponding to a large number of antennas at the FC demonstrates the rate at which the transmit gain of the sensors can be decreased upon increasing the number of antennas at the FC. This in turn has a significant implication for the WSN, since the reduced transmit power can lead to improved battery life of the sensors. 


\section{A. Known Parameter}

This subsection presents the asymptotic performance of the test statistic in (11) for both identical and optimal gain allocation.

1) Uniformly Distributed Gain Allocation: Under this scheme, equal power is allocated to all the sensors, i.e., $p_{u}=\left|f_{j}\right|^{2}=P / K, \forall j$. Let us consider the scaling of $p_{u}=\frac{\tilde{p}_{u}}{M}$, where $\tilde{p}_{u}$ denotes the average gain of each sensor. Using this scaling, the asymptotic performance of the detector in (11) in the large antenna regime can be derived as shown below.

Theorem 3. The asymptotic probabilities of detection and false alarm, represented by $P_{D}^{u}$ and $P_{F A}^{u}$, respectively, are given below for the test statistic $T_{K P}(\tilde{\mathbf{y}})$ of (11), as $M \rightarrow \infty$, and using identical gain allocation,

$$
P_{D}^{u}=Q\left(\gamma^{\prime}-\tilde{\mu}_{T_{K P} \mid \mathcal{H}_{1}}^{u}\right), P_{F A}^{u}=Q\left(\gamma^{\prime}\right),
$$

where the quantity $\tilde{\mu}_{T_{K P} \mid \mathcal{H}_{1}}^{u}$ represents the normalized mean corresponding to the alternative hypothesis and is given as

$$
\tilde{\mu}_{T_{K P} \mid \mathcal{H}_{1}}^{u}=\sqrt{\sum_{j=1}^{K} \frac{2 \tilde{p}_{u} \beta_{j}\left|a_{j}\right|^{2} \theta^{2}}{\sigma_{v}^{2} \tilde{p}_{u} \beta_{j}+\sigma_{n}^{2}}}
$$

Proof: See Appendix D.

2) Optimal Gain Allocation: To derive the asymptotic bounds on $P_{D}$ and $P_{F A}$ with optimal gain allocation as described in Section III-A, a modified KKT multiplier $\lambda^{\prime}$ is defined as $\lambda^{\prime}=\frac{\lambda}{M}$. Incorporating $\lambda^{\prime}$, the solution in (19) can be rewritten as

$$
p_{j}=\frac{1}{M}\left(\sqrt{\frac{\sigma_{n}^{2}\left|a_{j}\right|^{2}}{\lambda^{\prime} \beta_{j} \sigma_{v}^{4}}}-\frac{\sigma_{n}^{2}}{\beta_{j} \sigma_{v}^{2}}\right)^{+}=\frac{1}{M} \tilde{p}_{j},
$$

where $\sum_{j=1}^{K} \tilde{p}_{j}=P$. Similar to the previous analysis, $\lambda^{\prime}$ can be found via bisection search over $\left[\lambda_{l}^{\prime}, \lambda_{u}^{\prime}\right]$, where $\lambda_{l}^{\prime}$ and $\lambda_{u}^{\prime}$ denote the lower and upper bounds on $\lambda^{\prime}$, respectively, and are given as $\lambda_{l}^{\prime}=\min _{j} .\left\{\frac{\beta_{j} \sigma_{n}^{2}\left|a_{j}\right|^{2}}{\left(\beta_{j} \sigma_{v}^{2} P+\sigma_{n}^{2}\right)^{2}}\right\}$ and $\lambda_{u}^{\prime}=\max _{j} .\left\{\frac{\beta_{j}\left|a_{j}\right|^{2}}{\sigma_{n}^{2}}\right\}$. Following similar lines to those of the identical gain allocation, the asymptotic bounds on the corresponding $P_{D}$ and $P_{F A}$ values be obtained by using the scaling $p_{j}=\left|f_{j}\right|^{2}=\frac{\tilde{p}_{j}}{M}$ as described by the result below.

Theorem 4. The asymptotic probabilities of detection and false alarm, $P_{D}^{o}$ and $P_{F A}^{o}$, respectively, are determined below for the test $T_{K P}(\tilde{\mathbf{y}})$ in (11), as $M \rightarrow \infty$, in conjunction with optimal gain allocation,

$$
P_{D}^{o}=Q\left(\gamma^{\prime}-\tilde{\mu}_{T_{K P} \mid \mathcal{H}_{1}}^{o}\right), P_{F A}^{o}=Q\left(\gamma^{\prime}\right),
$$

where the normalized mean for $\mathcal{H}_{1}$, denoted by $\tilde{\mu}_{T_{K P} \mid \mathcal{H}_{1}}^{o}$, is given as

$$
\tilde{\mu}_{T_{K P} \mid \mathcal{H}_{1}}^{o}=\sqrt{\sum_{j=1}^{K} \frac{2 \tilde{p}_{j} \beta_{j}\left|a_{j}\right|^{2} \theta^{2}}{\sigma_{v}^{2} \tilde{p}_{j} \beta_{j}+\sigma_{n}^{2}}} .
$$

The proof of the above theorem follows similar lines to those of Theorem 3 with the scaling $p_{u}=\frac{\tilde{p}_{u}}{M}$ replaced by $p_{j}=\frac{\tilde{p}_{j}}{M}$

\section{B. Unknown Parameter}

Consider the scaling of $p_{u}=\frac{\tilde{p}_{u}}{M}$. The next result presents the asymptotic performance of the test in (23) for the detection of an unknown parameter in a massive MIMO WSN.

Theorem 5. The asymptotic probabilities of detection $P_{D}^{u}$ and false alarm $P_{F A}^{u}$ of the detector in (23), are given below for identical gain allocation,

$P_{D}^{u}=Q\left(\gamma^{\prime \prime}-\tilde{\mu}_{T_{U P}^{\prime} \mid \mathcal{H}_{1}}^{u}\right)+Q\left(\gamma^{\prime \prime}+\tilde{\mu}_{T_{U P}^{\prime} \mid \mathcal{H}_{1}}^{u}\right), P_{F A}^{u}=2 Q\left(\gamma^{\prime \prime}\right)$,

where $\gamma^{\prime \prime}$ represents the detection threshold and $\tilde{\mu}_{T_{U P}^{\prime} \mid \mathcal{H}_{1}}^{u}$ is the normalized mean for the hypothesis $\mathcal{H}_{1}$, which is obtained as

$$
\tilde{\mu}_{T_{U P}^{\prime} \mid \mathcal{H}_{1}}^{u}=\sqrt{\sum_{j=1}^{K} \frac{2 \tilde{p}_{u} \beta_{j}\left|a_{j}\right|^{2} \theta^{2}}{\sigma_{v}^{2} \tilde{p}_{u} \beta_{j}+\sigma_{n}^{2}}} .
$$

Proof: Follows lines similar to those of Theorem 3.

Finally, following a procedure similar to that of Theorem 5 above, the corresponding performance metrics, i.e., $P_{D}^{o}$ and $P_{F A}^{o}$, for the detection of an unknown parameter in the massive MIMO WSN with perfect CSI and optimal gain allocation can be derived by substituting $p_{j}=\left|f_{j}\right|^{2}=\frac{\tilde{p}_{j}}{M}$ in place of $p_{u}=$ $\left|f_{j}\right|^{2}=\frac{\tilde{p}_{u}}{M}$. The closed-form expression of the normalized mean for this framework is explicitly given below:-

$$
\tilde{\mu}_{T_{\mathrm{UP}}^{\prime} \mid \mathcal{H}_{1}}^{o}=\sqrt{\sum_{j=1}^{K} \frac{2 \beta_{j}\left|a_{j}\right|^{2} \tilde{p}_{j} \theta^{2}}{\sigma_{v}^{2} \beta_{j} \tilde{p}_{j}+\sigma_{n}^{2}}} .
$$

A common feature of all the tests for a massive antenna array at the FC having perfect CSI, as it becomes evident from the above investigation, is that the transmit gain of each sensor can be reduced proportionally to $1 / M$ without degrading the $P_{D}, P_{F A}$ performance. This is indeed one of the key benefits of a massive MIMO WSN, which can be exploited to achieve significant power savings, without compromising the sensing performance. The next section presents the proposed detectors along with their analysis for scenarios having imperfect CSI in a massive MIMO WSN.

\section{FRAMEWORK FOR IMPERFECT CSI}

Let $\tau$ pilot symbols be transmitted in each channel coherence interval of duration $\tau_{c}$, for channel estimation in the massive MIMO WSN. The remaining $\left(\tau_{c}-\tau\right)$ symbols are used for data transmission. All the sensors are assumed to simultaneously transmit orthogonal pilot sequences of length $\tau$ so that the resultant pilot matrix $\boldsymbol{\Phi} \in \mathbb{C}^{\tau \times K}$ is orthogonal, and satisfies the property $\boldsymbol{\Phi}^{H} \boldsymbol{\Phi}=\mathbf{I}_{K}$. The received signal matrix $\mathbf{Y}_{p} \in \mathbb{C}^{M \times \tau}$ at the FC corresponding to the transmitted pilot matrix can be expressed as

$$
\mathbf{Y}_{p}=\sqrt{p_{p}} \mathbf{G} \boldsymbol{\Phi}^{T}+\mathbf{W}_{p}
$$

where $p_{p}=\tau p_{u}$ is the pilot power, and $\mathbf{W}_{p} \in \mathbb{C}^{M \times \tau}$ denotes the noise matrix with i.i.d. elements distributed as $w_{p, i, j} \sim$ $\mathcal{C N}\left(0, \sigma_{w}^{2}\right)$. From (38), the MMSE estimate of $\mathbf{G}$ is [24]

$$
\hat{\mathbf{G}}=\frac{1}{\sqrt{p_{p}}} \mathbf{Y}_{p} \boldsymbol{\Phi}^{*}\left(\frac{\sigma_{w}^{2}}{p_{p}} \mathbf{D}^{-1}+\mathbf{I}_{K}\right)^{-1} .
$$


Let the corresponding estimation error matrix be defined as $\mathcal{E}=\hat{\mathbf{G}}-\mathbf{G}$. The $j$-th columns of $\hat{\mathbf{G}}$ and $\mathcal{E}$ denoted by $\hat{\mathbf{g}}_{j}$ and $\varepsilon_{j}$, respectively, follow the complex Gaussian distributions determined as $\hat{\mathbf{g}}_{j} \sim \mathcal{C N}\left(\mathbf{0}, \beta_{\hat{g}, j} \mathbf{I}_{M}\right), \beta_{\hat{g}, j} \triangleq p_{p} \beta_{j}^{2} /\left(\sigma_{w}^{2}+p_{p} \beta_{j}\right)$ and $\boldsymbol{\varepsilon}_{j} \sim \mathcal{C} \mathcal{N}\left(\mathbf{0}, \gamma_{\varepsilon, j} \mathbf{I}_{M}\right), \gamma_{\varepsilon, j} \triangleq \sigma_{w}^{2} \beta_{j} /\left(\sigma_{w}^{2}+p_{p} \beta_{j}\right)$. For the imperfect CSI scenario, the signal in (1) received at the FC under both the hypotheses can be modified as

$$
\begin{aligned}
& \mathcal{H}_{0}: \mathbf{y}=\hat{\mathbf{G}} \mathbf{F v}-\mathcal{E} \mathbf{F v}+\mathbf{n}, \\
& \mathcal{H}_{1}: \mathbf{y}=\hat{\mathbf{G}} \mathbf{F a} \theta-\mathcal{E} \mathbf{F a} \theta+\hat{\mathbf{G}} \mathbf{F v}-\mathcal{E} \mathbf{F v}+\mathbf{n} .
\end{aligned}
$$

Furthermore, upon performing MRC processing with the estimated channel matrix $\hat{\mathbf{G}}$ at the receiver and using the property $\frac{1}{M} \hat{\mathbf{G}}^{H} \hat{\mathbf{G}} \approx \mathbf{D}_{\hat{\mathrm{g}}}$, where $\mathbf{D}_{\hat{\mathrm{g}}}=\operatorname{diag}\left(\beta_{\hat{g}, 1}, \ldots, \beta_{\hat{g}, K}\right)$ [26], the output signals corresponding to both the hypotheses in (40) reduce to

$$
\begin{aligned}
& \mathcal{H}_{0}: \breve{\mathbf{y}} \approx \mathbf{w}, \\
& \mathcal{H}_{1}: \breve{\mathbf{y}} \approx \mathbf{D}_{\hat{\mathbf{g}}} \mathbf{F a} \theta-\frac{1}{M} \hat{\mathbf{G}}^{H} \mathcal{E} \mathbf{F a} \theta+\mathbf{w},
\end{aligned}
$$

where $\mathbf{w}=\mathbf{D}_{\hat{\mathbf{g}}} \mathbf{F} \mathbf{v}-\frac{1}{M} \hat{\mathbf{G}}^{H} \mathcal{E} \mathbf{F} \mathbf{v}+\frac{1}{M} \hat{\mathbf{G}}^{H} \mathbf{n} \in \mathbb{C}^{K \times 1}$ denotes the equivalent noise vector that has the covariance matrix of $\mathbf{C}_{\mathbf{w}}=\sigma_{v}^{2} \mathbf{D}_{\hat{\mathrm{g}}} \mathbf{F} \mathbf{F}^{H} \mathbf{D}_{\hat{\mathrm{g}}}^{H}+\frac{\sigma_{v}^{2}}{M}\left(\sum_{j=1}^{K} \gamma_{\varepsilon, j}\left|f_{j}\right|^{2}\right) \mathbf{D}_{\hat{\mathrm{g}}}+\frac{\sigma_{n}^{2}}{M} \mathbf{D}_{\hat{\mathrm{g}}}$. The imperfect CSI analysis is inspired by other contribution on massive MIMO, especially by [26], which demonstrates the SINR and power scaling laws. Their analysis shows that the implications for imperfect CSI and the pertinent analysis is significantly different from that of the perfect CSI case. Now the detectors having CSI uncertainty for the known and unknown parameter scenarios are derived below.

\section{A. Fusion Rule for Known $\theta$ with Imperfect CSI}

The distributions of the output $\breve{\mathbf{y}}$ corresponding to both the hypotheses, for this scenario, can be derived as

$$
\begin{aligned}
& \mathcal{H}_{0}: \breve{\mathbf{y}} \stackrel{a}{\sim} \mathcal{C N}\left(\mathbf{0}, \mathbf{C}_{\mathbf{w}}\right), \\
& \mathcal{H}_{1}: \breve{\mathbf{y}} \stackrel{a}{\sim} \mathcal{C} \mathcal{N}\left(\mathbf{D}_{\hat{\mathbf{g}}} \mathbf{F a} \theta, \mathbf{C}_{\boldsymbol{\eta}}\right),
\end{aligned}
$$

where $\mathbf{C}_{\boldsymbol{\eta}}=\mathbf{C}_{\mathbf{s}}+\mathbf{C}_{\mathbf{w}}$ denotes the equivalent noise covariance matrix under hypothesis $\mathcal{H}_{1}$ with $\mathbf{C}_{\mathbf{s}} \in \mathbb{C}^{K \times K}$ given as $\mathbf{C}_{\mathbf{s}}=$ $\frac{1}{M}\left(\sum_{j=1}^{K} \gamma_{\varepsilon, j}\left|a_{j}\right|^{2}\left|f_{j}\right|^{2}\right) \mathbf{D}_{\hat{\mathbf{g}}}$. The LLR test $T_{\mathrm{KIP}}(\breve{\mathbf{y}})$ for the filtered output in (41) for the detection of the parameter $\theta$ in a massive MIMO WSN with imperfect CSI can be formulated as

$$
\begin{aligned}
& T_{\mathrm{KIP}}(\breve{\mathbf{y}})=\ln \left[\frac{p\left(\breve{\mathbf{y}} \mid \mathcal{H}_{1}\right)}{p\left(\breve{\mathbf{y}} \mid \mathcal{H}_{0}\right)}\right] \\
& =\breve{\mathbf{y}}^{H} \mathbf{C}_{\mathbf{w}}^{-1} \breve{\mathbf{y}}-\left(\breve{\mathbf{y}}-\mathbf{D}_{\hat{\mathbf{g}}} \mathbf{F a} \theta\right)^{H} \mathbf{C}_{\boldsymbol{\eta}}^{-1}\left(\breve{\mathbf{y}}-\mathbf{D}_{\hat{\mathbf{g}}} \mathbf{F a} \theta\right) \\
& =\breve{\mathbf{y}}^{H}\left(\mathbf{C}_{\mathbf{w}}^{-1}-\mathbf{C}_{\boldsymbol{\eta}}^{-1}\right) \breve{\mathbf{y}}+2 \Re\left(\theta \mathbf{a}^{H} \mathbf{F}^{H} \mathbf{D}_{\hat{\mathbf{g}}}^{H} \mathbf{C}_{\boldsymbol{\eta}}^{-1} \breve{\mathbf{y}}\right) .
\end{aligned}
$$

Upon substituting all the matrices and vectors in the above expression, the test statistic in (43) reduces to

$$
\begin{aligned}
T_{\mathrm{KIP}}(\breve{\mathbf{y}}) & =\sum_{j=1}^{K} \frac{\sigma_{s, j}^{2}\left|\breve{y}_{j}\right|^{2}}{\sigma_{w, j}^{2} \sigma_{\eta, j}^{2}}+2 \Re\left(\sum_{j=1}^{K} \frac{\theta a_{j}^{*} f_{j}^{*} \beta_{\hat{g}, j}^{*} \breve{y}_{j}}{\sigma_{\eta, j}^{2}}\right) \\
& =\sum_{j=1}^{K}\left[\alpha_{j}\left|\breve{y}_{j}\right|^{2}+2 \Re\left(\frac{\theta \alpha_{j} a_{j}^{*} f_{j}^{*} \beta_{\hat{g}, j}^{*} \sigma_{w, j}^{2} \breve{y}_{j}}{\sigma_{s, j}^{2}}\right)\right] \\
& =\sum_{j=1}^{K} \alpha_{j}\left[\left(\breve{y}_{j}+\delta_{j}\right)^{*}\left(\breve{y}_{j}+\delta_{j}\right)-\delta_{j}^{2}\right]
\end{aligned}
$$

where the parameters $\alpha_{j}, \delta_{j}$ are defined as $\alpha_{j}=\frac{\sigma_{s, j}^{2}}{\sigma_{w, j}^{2} \sigma_{\eta, j}^{2}}$ and $\delta_{j}=\frac{\theta a_{j}^{*} f_{j}^{*} \beta_{\hat{g}, j}^{*} \sigma_{w, j}^{2}}{\sigma_{s, j}^{2}}$, respectively. The test statistic in (44) can be further simplified as

$$
T_{\mathrm{KIP}}(\breve{\mathbf{y}})=\sum_{j=1}^{K} \underbrace{\alpha_{j}\left|\breve{y}_{j}+\delta_{j}\right|^{2}}_{T_{\mathrm{KIP}}\left(\breve{y}_{j}\right)}=\mathbf{y}_{\mathrm{I}}^{H} \boldsymbol{\alpha} \mathbf{y}_{\mathrm{I}} \underset{\mathcal{H}_{0}}{\stackrel{\mathcal{H}_{1}}{\gtrless}} \tilde{\gamma},
$$

where the vector $\mathbf{y}_{\mathrm{I}} \in \mathbb{C}^{K \times 1}$ and the matrix $\boldsymbol{\alpha} \in \mathbb{C}^{K \times K}$ are defined as $\mathbf{y}_{\mathrm{I}}=\left[\left(\breve{y}_{1}+\delta_{1}\right), \ldots,\left(\breve{y}_{K}+\delta_{K}\right)\right]^{T}$ and $\boldsymbol{\alpha}=$ $\operatorname{diag}\left(\alpha_{1}, \ldots, \alpha_{K}\right)$, respectively. From (45), the PDFs of the component test statistic $T_{\mathrm{KIP}}\left(\breve{y}_{j}\right)$ under both the hypotheses are given as

$$
\begin{aligned}
& \mathcal{H}_{0}: T_{\mathrm{KIP}}\left(\breve{y}_{j}\right)=\frac{\alpha_{j} \sigma_{w, j}^{2}}{2} \frac{\left|\breve{y}_{j}+\delta_{j}\right|^{2}}{\sigma_{w, j}^{2} / 2}=\frac{\lambda_{j, 0}}{2} \chi_{2}^{\prime 2}\left(\xi_{j, 0}\right), \\
& \mathcal{H}_{1}: T_{\mathrm{KIP}}\left(\breve{y}_{j}\right)=\frac{\alpha_{j} \sigma_{\eta, j}^{2}}{2} \frac{\left|\breve{y}_{j}+\delta_{j}\right|^{2}}{\sigma_{\eta, j}^{2} / 2}=\frac{\lambda_{j, 1}}{2} \chi_{2}^{\prime 2}\left(\xi_{j, 1}\right),
\end{aligned}
$$

where $\chi_{2}^{\prime 2}\left(\xi_{j, 0}\right), \chi_{2}^{\prime 2}\left(\xi_{j, 1}\right)$ denote non-central chi-squared distributed random variables with two degrees of freedom and $\xi_{j, 0}=\frac{2 \delta_{j}^{2}}{\sigma_{w, j}^{2}}, \xi_{j, 1}=\frac{2\left(\theta a_{j} f_{j} \beta_{\hat{g}, j}+\delta_{j}\right)^{2}}{\sigma_{\eta, j}^{2}}$ denote the non-centrality parameters, respectively, with $\lambda_{j, 0}=\alpha_{j} \sigma_{w, j}^{2}$ and $\lambda_{j, 1}=$ $\alpha_{j} \sigma_{\eta, j}^{2}$. Using (46), the test statistic $T_{\mathrm{KIP}}(\breve{\mathbf{y}})$ can be equivalently represented as the weighted sum of chi-squared random variables with two degrees of freedom as shown below:-

$$
\begin{aligned}
& \mathcal{H}_{0}: T_{\mathrm{KIP}}(\breve{\mathbf{y}})=\sum_{j=1}^{K} T_{\mathrm{KIP}}\left(\breve{y}_{j}\right) \equiv \sum_{j=1}^{K} \frac{\lambda_{j, 0}}{2} \chi_{2}^{\prime 2}\left(\xi_{j, 0}\right), \\
& \mathcal{H}_{1}: T_{\mathrm{KIP}}(\breve{\mathbf{y}})=\sum_{j=1}^{K} T_{\mathrm{KIP}}\left(\breve{y}_{j}\right) \equiv \sum_{j=1}^{K} \frac{\lambda_{j, 1}}{2} \chi_{2}^{\prime 2}\left(\xi_{j, 1}\right) .
\end{aligned}
$$

The test statistic $T_{\mathrm{KIP}}(\breve{\mathbf{y}})$ under both the hypotheses can be closely approximated as the non-central chi-squared random variables

$$
\begin{aligned}
& \mathcal{H}_{0}: T_{\mathrm{KIP}}(\breve{\mathbf{y}}) \approx \chi_{l_{\mathrm{KF}}}^{\prime 2}\left(\xi_{\mathrm{KF}}\right), \\
& \mathcal{H}_{1}: T_{\mathrm{KIP}}(\breve{\mathbf{y}}) \approx \chi_{l_{\mathrm{KD}}}^{\prime 2}\left(\xi_{\mathrm{KD}}\right),
\end{aligned}
$$

where the degrees of freedom, i.e., $l_{\mathrm{KF}}, l_{\mathrm{KD}}$ and the noncentrality parameters, i.e., $\xi_{\mathrm{KF}}, \xi_{\mathrm{KD}}$, of the chi-squared random variables are obtained from the first four cumulants of $T_{\mathrm{KIP}}(\breve{\mathbf{y}})$ using the result in [41]. Employing (48), the closed-form expressions of $P_{D}$ and $P_{F A}$ for the test statistic in (45) can be determined as

$$
\begin{aligned}
P_{D} & \approx \operatorname{Pr}\left(\chi_{l_{\mathrm{KD}}}^{\prime 2}\left(\xi_{\mathrm{KD}}\right)>\tilde{\gamma}\right)=Q_{\chi_{l_{\mathrm{KD}}}^{\prime 2}\left(\xi_{\mathrm{KD}}\right)}(\tilde{\gamma}), \\
P_{F A} & \approx \operatorname{Pr}\left(\chi_{l_{\mathrm{KF}}}^{\prime 2}\left(\xi_{\mathrm{KF}}\right)>\tilde{\gamma}\right)=Q_{\chi_{l_{\mathrm{KF}}^{\prime}}^{\prime 2}\left(\xi_{\mathrm{KF}}\right)}(\tilde{\gamma}) .
\end{aligned}
$$

The expressions in (49) and (50) can be further simplified by exploiting the asymptotic property of the chi-squared random variable [37] as described in the theorem below.

Theorem 6. For a given threshold $\tilde{\gamma}$, the probabilities of detection $P_{D}$ and false alarm $P_{F A}$ for the proposed detector in (45), can be approximated as seen below for a massive MIMO setup with a large number of antennas $M$ at the FC and imperfect CSI,

$$
P_{D} \approx Q\left(\frac{\tilde{\gamma}-\mu_{K D \mid \mathcal{H}_{1}}}{\sigma_{K D \mid \mathcal{H}_{1}}}\right), P_{F A} \approx Q\left(\frac{\tilde{\gamma}-\mu_{K F \mid \mathcal{H}_{0}}}{\sigma_{K F \mid \mathcal{H}_{0}}}\right),
$$


where the moments of the test statistic $T_{K I P}(\breve{\mathbf{y}})$ under the two hypotheses can be derived as

$$
\begin{gathered}
\mathbb{E}\left\{T_{K I P}(\breve{\mathbf{y}}) ; \mathcal{H}_{0}\right\}=\mu_{K F \mid \mathcal{H}_{0}}=l_{K F}+\xi_{K F}, \\
\mathbb{E}\left\{T_{K I P}(\breve{\mathbf{y}}) ; \mathcal{H}_{1}\right\}=\mu_{K D \mid \mathcal{H}_{1}}=l_{K D}+\xi_{K D}, \\
\operatorname{var}\left\{T_{K I P}(\breve{\mathbf{y}}) ; \mathcal{H}_{0}\right\}=\sigma_{K F \mid \mathcal{H}_{0}}^{2}=2 l_{K F}+4 \xi_{K F}, \\
\operatorname{var}\left\{T_{K I P}(\breve{\mathbf{y}}) ; \mathcal{H}_{1}\right\}=\sigma_{K D \mid \mathcal{H}_{1}}^{2}=2 l_{K D}+4 \xi_{K D},
\end{gathered}
$$

Proof: See Appendix E.

\section{B. Fusion Rule for Unknown $\theta$ with Imperfect CSI}

This section considers a general scenario for the detection of an unknown parameter with imperfect CSI at the FC. The corresponding GLRT based test statistic can be formulated as

$$
T_{\text {UIP }}(\breve{\mathbf{y}})=\ln \left[\frac{p\left(\breve{\mathbf{y}} ; \hat{\theta} \mid \mathcal{H}_{1}\right)}{p\left(\breve{\mathbf{y}} \mid \mathcal{H}_{0}\right)}\right] .
$$

The PDFs of the filter output $\breve{\mathbf{y}}$ under hypotheses $\mathcal{H}_{0}$ and $\mathcal{H}_{1}$, parameterized by $\hat{\theta}$, can be expressed as

$$
\begin{aligned}
& p\left(\breve{\mathbf{y}} \mid \mathcal{H}_{0}\right)=\frac{1}{\pi^{K}\left|\mathbf{C}_{\mathbf{w}}\right|} \exp \left(-\breve{\mathbf{y}}^{H} \mathbf{C}_{\mathbf{w}}^{-1} \breve{\mathbf{y}}\right), \\
& p\left(\breve{\mathbf{y}} ; \hat{\theta} \mid \mathcal{H}_{1}\right)=\frac{1}{\pi^{K}\left|\mathbf{C}_{\boldsymbol{\eta}}\right|} \exp \left(-\left\|\breve{\mathbf{y}}-\mathbf{D}_{\hat{\mathbf{g}}} \mathbf{F a} \hat{\theta}\right\|_{\mathbf{C}_{\boldsymbol{\eta}}^{-1}}^{2}\right) .
\end{aligned}
$$

Substituting the above PDFs in (56), the desired test statistic $T_{\text {UIP }}(\breve{\mathbf{y}})$ can be determined as

$$
\begin{aligned}
T_{\mathrm{UIP}}(\breve{\mathbf{y}}) & =\breve{\mathbf{y}}^{H}\left(\mathbf{C}_{\mathbf{w}}^{-1}-\mathbf{C}_{\boldsymbol{\eta}}^{-1}\right) \breve{\mathbf{y}}+2 \Re\left(\breve{\mathbf{y}}^{H} \mathbf{C}_{\boldsymbol{\eta}}^{-1} \mathbf{D}_{\hat{\mathrm{g}}} \mathbf{F a} \hat{\theta}\right) \\
& -\hat{\theta}^{2} \mathbf{a}^{H} \mathbf{F}^{H} \mathbf{D}_{\hat{\mathrm{g}}}^{H} \mathbf{C}_{\boldsymbol{\eta}}^{-1} \mathbf{D}_{\hat{\mathrm{g}}} \mathbf{F a},
\end{aligned}
$$

where $\hat{\theta}$ represents the ML estimate of the parameter $\theta$ under hypothesis $\mathcal{H}_{1}$, which can be obtained by maximizing $p\left(\breve{\mathbf{y}} ; \theta \mid \mathcal{H}_{1}\right)$ as $\hat{\theta}=\frac{\Re\left(\mathbf{a}^{H} \mathbf{F}^{H} \mathbf{D}_{\hat{\mathbf{g}}}^{H} \mathbf{C}_{\boldsymbol{\eta}}^{-1} \breve{\mathbf{y}}\right)}{\mathbf{a}^{H} \mathbf{F}^{H} \mathbf{D}_{\hat{\mathbf{g}}}^{H} \mathbf{C}_{\eta}^{-1} \mathbf{D}_{\hat{\mathbf{g}} \mathbf{F a}}}$ [40]. Substituting $\hat{\theta}$ in (58), the test statistic $T_{\mathrm{UIP}}(\hat{\mathbf{y}})$ can be simplified as

$$
T_{\mathrm{UIP}}(\breve{\mathbf{y}})=\breve{\mathbf{y}}^{H}\left(\mathbf{C}_{\mathbf{w}}^{-1}-\mathbf{C}_{\boldsymbol{\eta}}^{-1}\right) \breve{\mathbf{y}}+\frac{\left[\Re\left(\mathbf{a}^{H} \mathbf{F}^{H} \mathbf{D}_{\hat{\mathrm{g}}}^{H} \mathbf{C}_{\boldsymbol{\eta}}^{-1} \breve{\mathbf{y}}\right)\right]^{2}}{\mathbf{a}^{H} \mathbf{F}^{H} \mathbf{D}_{\hat{\mathrm{g}}}^{H} \mathbf{C}_{\boldsymbol{\eta}}^{-1} \mathbf{D}_{\hat{\mathrm{g}}} \mathbf{F a}} \underset{\mathcal{H}_{0}}{\gtrless} \tilde{\gamma}^{\prime} .
$$

The above test can now be used directly for detection purposes. However, the characterization of its detection performance is mathematically intractable. Therefore, in order to evaluate the performance, the test statistic in (59) can be approximated as

$$
\begin{aligned}
T_{\mathrm{UIP}}(\breve{\mathbf{y}}) & \approx \breve{\mathbf{y}}^{H}\left[\left(\mathbf{C}_{\mathbf{w}}^{-1}-\mathbf{C}_{\boldsymbol{\eta}}^{-1}\right)+\frac{\mathbf{C}_{\boldsymbol{\eta}}^{-1} \mathbf{D}_{\hat{\mathrm{g}}} \mathbf{F a a}{ }^{H} \mathbf{F}^{H} \mathbf{D}_{\hat{\mathrm{g}}}^{H} \mathbf{C}_{\boldsymbol{\eta}}^{-1}}{\mathbf{a}^{H} \mathbf{F}^{H} \mathbf{D}_{\hat{\mathrm{g}}}^{H} \mathbf{C}_{\boldsymbol{\eta}}^{-1} \mathbf{D}_{\hat{\mathrm{g}} \mathbf{F}} \mathbf{a}}\right] \breve{\mathbf{y}} \\
& =\breve{\mathbf{y}}^{H} \mathbf{X} \breve{\mathbf{y}},
\end{aligned}
$$

where the matrix $\mathbf{X}_{H^{-1}}^{\text {can }}$ be defined as $\mathbf{X}=$ $\left(\mathbf{C}_{\mathbf{w}}^{-1}-\mathbf{C}_{\boldsymbol{\eta}}^{-1}\right)+\frac{\mathbf{C}_{\boldsymbol{\eta}}^{-1} \mathbf{D}_{\hat{\mathrm{g}}} \mathbf{F a a}{ }^{H} \mathbf{F}^{H} \mathbf{D}_{\hat{\mathbf{g}}}^{H} \mathbf{C}_{\boldsymbol{\eta}}^{-1}}{\mathbf{a}^{H} \mathbf{F}^{H} \mathbf{D}_{\hat{\mathrm{g}}}^{H} \mathbf{C}_{\boldsymbol{\eta}}^{-1} \mathbf{D}_{\hat{\mathrm{g}}} \mathbf{F a}}$. The performance of the test statistic above is described in the theorem given below.

Theorem 7. The probabilities of detection $P_{D}$ and false alarm $P_{F A}$ for the test statistic in (60), corresponding to the detection of an unknown parameter at the FC for imperfect CSI, are given as

$$
P_{D} \approx Q_{\chi_{l_{U D}}^{\prime 2}\left(\xi_{U D}\right)}\left(\tilde{\gamma}^{\prime}\right), P_{F A} \approx Q_{\chi_{l_{U F}^{\prime}}^{\prime 2}\left(\xi_{U F}\right)}\left(\tilde{\gamma}^{\prime}\right),
$$

where the quantities $l_{U F}, l_{U D}$ and $\xi_{U F}, \xi_{U D}$ represent the degree of freedom and the non-centrality parameter of the chi-squared random variables $\chi_{l_{U F}}^{\prime 2}\left(\xi_{U F}\right)$ and $\chi_{l_{U D}}^{\prime 2}\left(\xi_{U D}\right)$, respectively, as described in Appendix $F$.

\section{Simulation Results}

In this section our simulation results are presented for characterizing the performance of the proposed schemes for correlated parameter detection in massive MIMO WSNs operating in different scenarios. For our simulations, a total of $K=12$ sensors are deployed randomly with a uniform distribution around the FC in a disc-shaped region determined by the maximum and minimum radii of $r_{m}=1000$ meters and $r_{h}=100$ meters, respectively. Such a system can be deployed in practice for environmental and agricultural monitoring applications, to sense various parameters of interest such as temperature, pressure, humidity, soil moisture etc. Consequently, the sensor decisions are potentially correlated, since the sensors are densely located and observe the same phenomenon. The large-scale fading matrix $\mathbf{D}$ is generated similar to [26], i.e. the large-scale fading coefficients are modeled as $\beta_{j}=z_{j} /\left(r_{j} / r_{h}\right)^{\nu}$, where $z_{j}$ is a log-normal random variable with a mean of $\mu_{s}=3$ and standard deviation of $\sigma_{\mathrm{s}}=8 \mathrm{~dB}, r_{j}$ denotes the distance of the $j$-th sensor from the FC and $\nu$ represents the path-loss exponent that is chosen as $\nu=3.8$. The correlation coefficient $a_{j}, 1 \leq j \leq K$, is assumed to be uniformly distributed between $[0.5,0.9]$. Moreover, the parameters such as the observation noise power, receiver noise power, total sensor transmit gain and number of FC antennas are set as $\sigma_{v}^{2}=0.2, \sigma_{n}^{2}=0.7, P=1$ and $M=50$, respectively. Hence, the SNRs $P / \sigma_{v}^{2}$ and $P / \sigma_{n}^{2}$ are set as $6.99 \mathrm{~dB}, 1.55 \mathrm{~dB}$, respectively. In the following plots, each point is obtained as a result of averaging over 100, 000 trials.

Fig. 3a compares the receiver operating characteristic (ROC) of the proposed detector in (11) to that of the detector proposed in [11]. The authors in [11] consider an amplify and forward scheme for transmitting the sensor observations to the FC, which is assumed to be equipped with multiple antennas, but not a massive MIMO antenna array. Furthermore, the work therein does not consider a correlated parameter but an identical parameter $\theta$ across all the sensors. Hence, for a fair comparison with the scheme in [11], the spatial correlation vector $\mathbf{a}$ is set as $\mathbf{a}=\mathbf{1}$. It is also worth noting that the analysis therein is limited only to the error exponent, but does not consider the actual probabilities of detection or false alarm. The number of FC antennas for the detector in [11] is set to $M=\{3,10,50\}$ and the other parameters are kept the same. It is observed that the proposed low-complexity scheme is only suitable for a massive MIMO system, which has a similar performance as the detector in [11] using $M=50$. This illustrates the advantage of employing a large antenna array. Moreover, the performance of the proposed scheme further improves upon utilizing the optimal sensor gains derived in Section III-A. Similarly, Fig. 3b demonstrates the performance of the detector proposed in (23) for the uniform and optimal gain allocation schemes. The performance of the 


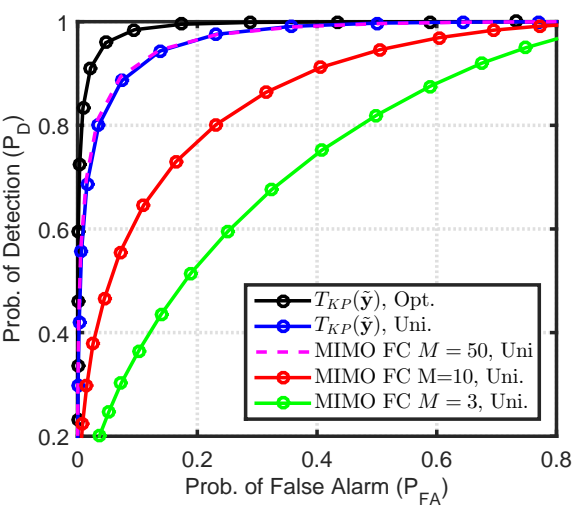

(a)

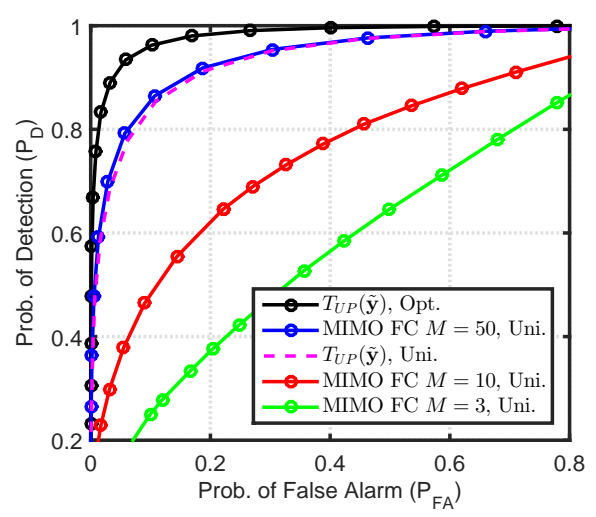

(b)

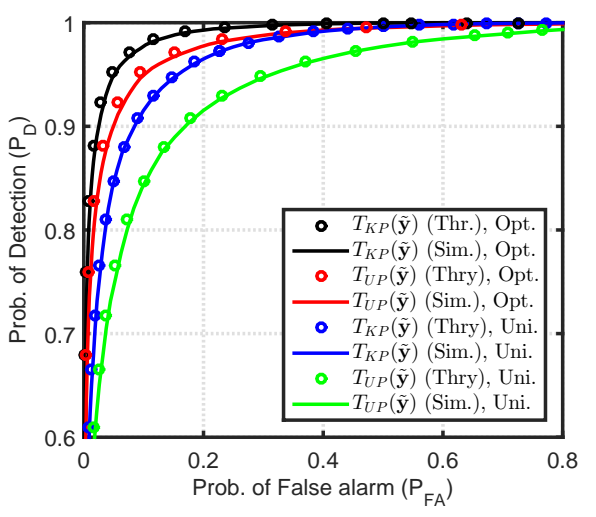

(c)

Fig. 3. ROC plot for comparing (a) the detector $T_{\mathrm{KP}}(\tilde{\mathbf{y}})$ in (11) using $M=50$ with the detectors in [11] using $M=\{3,10,50\}$ for $K=12, \sigma_{v}^{2}=0.2$, $\sigma_{n}^{2}=0.7$ and $P=1$. (b) the detector $T_{\mathrm{UP}}(\tilde{\mathbf{y}})$ in (23) using $M=50$ with GLRT detectors using $M=\{3,10,50\}$ for $K=12, \sigma_{v}^{2}=0.2, \sigma_{n}^{2}=0.7$ and $P=1$. (c) the simulated plots of the detectors in (11) and (23) with their theoretical results in Theorem 1 and Theorem 2 , respectively, for $K=12$, $M=100, \sigma_{v}^{2}=0.2, \sigma_{n}^{2}=0.7$ and $P=1$.

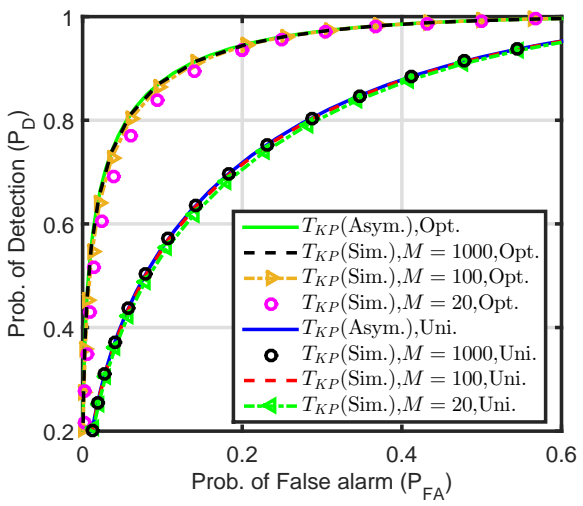

(a)

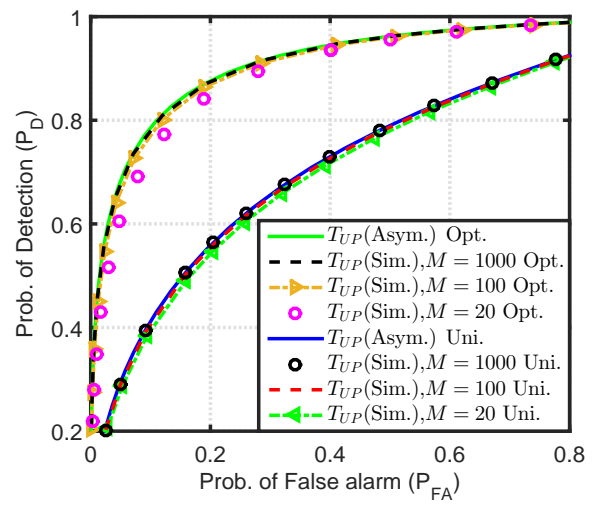

(b)

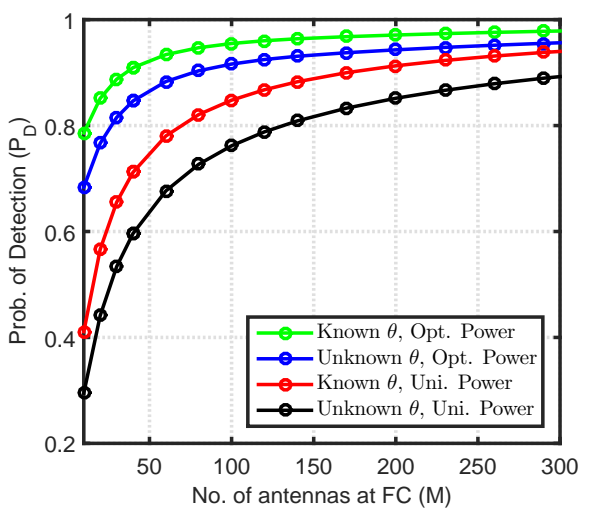

(c)

Fig. 4. For uniform and optimized gain allocation schemes, (a) large antenna performance analysis for the detector $T_{\mathrm{KP}}(\tilde{\mathbf{y}})$ in (11) for a WSN with $K=12$, $\sigma_{v}^{2}=0.2, \sigma_{n}^{2}=0.7, P=10$ and $M=\{20,100,1000\}$. (b) large antenna performance analysis for the detector $T_{\mathrm{UP}}(\tilde{\mathbf{y}})$ in (23) for a WSN with $K=12$, $\sigma_{v}^{2}=0.2, \sigma_{n}^{2}=0.7, P=10$ and $M=\{20,100,1000\}$. (c) $P_{D}$ vs. $M$ for the detectors $T_{\mathrm{KP}}(\tilde{\mathbf{y}})$ in $(11)$ and $T_{\mathrm{UP}}(\tilde{\mathbf{y}})$ in $(23)$, with $K=12, \sigma_{v}^{2}=0.2$, $\sigma_{n}^{2}=0.7$ and $P=1$.

GLRT directly using $\mathbf{y}$ in (1), without employing linear preprocessing at the FC is also shown. For this plot, the number of antennas at the FC is set to $M \in\{3,10,50\}$. It can be concluded from the figure that a similar detection performance can be achieved by the proposed low-complexity detector as that of the GLRT based MIMO detector for $M=50$ antennas. This demonstrates the efficiency of using the low-complexity MRC pre-filtering at the massive MIMO FC in the large antenna regime. Fig. 3c compares the analytical values of the probabilities of detection and false alarm, obtained using the expressions derived in Theorem 1 and Theorem 2 for the detectors in (11) and (23), respectively, with their simulation counterparts. As the processing step in (6) assumes a large number of antennas at the massive MIMO FC, the simulated and analytical results coincide for large values of $M(\sim 100)$, as it can be seen from the figure.

Fig. $4 \mathrm{a}$ and $4 \mathrm{~b}$ demonstrate the large array performance for the detectors in (11) and (23), respectively, for perfect CSI. For the system using identical power allocation, the gains are scaled as $p_{u}=\frac{\tilde{p}_{u}}{M}$, while the scaling is chosen as $p_{j}=\frac{\tilde{p}_{j}}{M}$ for optimal gain allocation. It can be readily

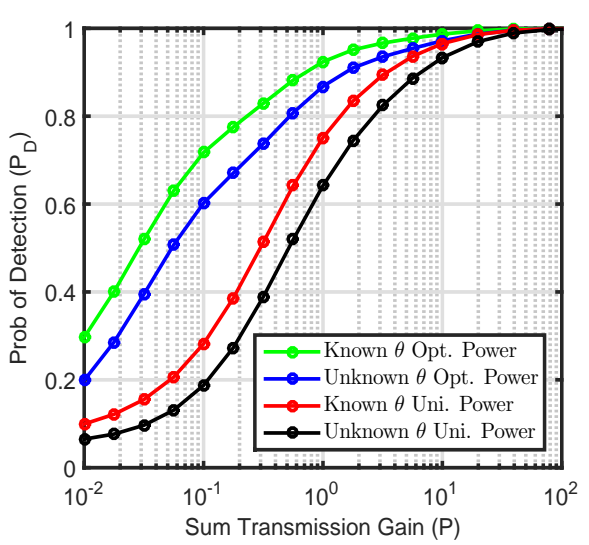

Fig. 5. $P_{D}$ vs. sum transmission gain $P$ for a WSN with $K=12, M=50$, $\sigma_{v}^{2}=0.2, \sigma_{n}^{2}=0.7$ and $P_{F A}=0.05$.

inferred from the figures that the simulated ROC plots of the proposed detectors approach their respective analytical plots, obtained using the expressions derived in Section V, as $M$ becomes large. The parameters used for generating these plots are set as $P=10, M=\{20,100,1000\}$ and the rest of the parameters are kept unchanged. Fig. 4c demonstrates 
the performance improvement in terms of the probability of detection $\left(P_{D}\right)$ both for identical and optimal gain allocation for an increasing number of antennas $M$ at the $\mathrm{FC}$, with the sum of transmission gains set as $P=1$. A significant improvement can be observed for the optimal gain allocation scheme in comparison to the identical gain allocation scheme for both the detection schemes.

Fig. 5 shows the variation of the probability of detection $\left(P_{D}\right)$ vs. the total sensor transmit power $(P)$ for $P_{F A}=0.05$. It is evident from the figure that for $P=10 \mathrm{~dB}$, the probability of detection is higher than 0.9 and it is close to unity for $P=20 \mathrm{~dB}$. This demonstrates the significant performance improvement that can be achieved by optimal gain allocation, when utilized for the detection of both known and unknown parameters. Fig. 6a plots the ROC of the proposed detector in (11) and the original detector in (5) to characterize the performance. Moreover, it demonstrates a similar performance comparison for the unknown parameter scenario. Fig. $6 \mathrm{~b}$ plots the probability of detection $\left(P_{D}\right)$ versus the probability of false alarm $\left(P_{F A}\right)$ for the detectors in (45) and (59) corresponding to the known and unknown parameter detection scenarios in the presence of imperfect CSI, respectively. It can be observed from the figure that the simulated plots are in close agreement with the theoretical results obtained in Theorem 6 and Theorem 7 , thus, validating the analytical findings for the equal power allocation scheme. Fig. 6c plots the probability of detection $\left(P_{D}\right)$ versus the probability of false alarm $\left(P_{F A}\right)$ for the detectors $T_{\mathrm{KIP}}(\breve{\mathbf{y}})$ in (45), $T_{\mathrm{KIP}}(\mathbf{y})$ corresponding to the known parameter scenario, and the detector $T_{\text {UIP }}(\breve{y})$ in (59), as well as its simplification in (60) for the unknown parameter scenario with imperfect CSI. It can be observed that the performance of the test $T_{\mathrm{KIP}}(\breve{\mathbf{y}})$ coincides with $T_{\mathrm{KIP}}(\mathbf{y})$ for large values of $M(\sim=50)$. Furthermore, the plot also demonstrates the closeness of the rules $T_{\mathrm{UIP}}(\breve{\mathbf{y}})$ and $T_{\mathrm{UIP}}(\mathbf{y})$.

\section{CONCLUSIONS}

Powerful detectors were proposed for correlated parameter detection in a massive MIMO WSN. The NP criterion and GLRT based fusion rules were initially determined for the detection of known and unknown deterministic parameters, respectively, for perfect CSI at the FC. Closed-form expressions of probabilities of detection $\left(P_{D}\right)$ and false alarm $\left(P_{F A}\right)$ were determined for characterizing the performance of the proposed detectors. Furthermore, the optimal sensor transmit gains were derived utilizing the deflection coefficient framework to further enhance the detection performance of the proposed tests. The asymptotic detection performance and pertinent gain scaling laws were also derived for both uniform and optimal gain allocation for a large antenna array at the massive MIMO FC. The framework was further extended to a scenario having CSI uncertainty. Simulation results were presented to validate the theoretical findings and to demonstrate the improved detection performance of the proposed schemes. In future research, this framework can be extended to a scenario with multiple FCs, each equipped with a very large antenna array, to present the fusion rules and pertinent analysis considering the effects of pilot contamination.

\section{APPENDIX A}

\section{Proof of THEOREM 1}

The mean of the test statistic $T_{\mathrm{KP}}(\tilde{\mathbf{y}})$ in (11) under the alternative hypothesis $\mathcal{H}_{1}$, i.e., $\mu_{T_{\mathrm{KP}} \mid \mathcal{H}_{1}}$, is given by

$$
\begin{aligned}
\mu_{T_{\mathrm{KP}} \mid \mathcal{H}_{1}} & =\mathbb{E}\left\{\sum_{j=1}^{K} \Re\left(\frac{M a_{j}^{*} f_{j}^{*} \tilde{y}_{j}}{\sigma_{v}^{2} M \beta_{j}\left|f_{j}\right|^{2}+\sigma_{n}^{2}}\right) \mid \mathcal{H}_{1}\right\} \\
& =\sum_{j=1}^{K} \Re\left(\frac{M a_{j}^{*} f_{j}^{*} \mathbb{E}\left\{\tilde{y}_{j} \mid \mathcal{H}_{1}\right\}}{\sigma_{v}^{2} M \beta_{j}\left|f_{j}\right|^{2}+\sigma_{n}^{2}}\right),
\end{aligned}
$$

where the quantity $\mathbb{E}\left\{\tilde{y}_{j} \mid \mathcal{H}_{1}\right\}$ can be derived as

$$
\mathbb{E}\left\{\tilde{y}_{j} \mid \mathcal{H}_{1}\right\}=\mathbb{E}\left\{\beta_{j} f_{j} a_{j} \theta+\beta_{j} f_{j} v_{j}+\frac{1}{M} \mathbf{g}_{j}^{H} \mathbf{n}\right\}=\beta_{j} f_{j} a_{j} \theta .
$$

Substituting the above equation into (62), the mean $\mu_{T_{\mathrm{KP}} \mid \mathcal{H}_{1}}$ can be expressed as

$$
\mu_{T_{\mathrm{KP} \mid \mathcal{H}}}=\sum_{j=1}^{K} \Re\left(\frac{M \beta_{j}\left|a_{j}\right|^{2}\left|f_{j}\right|^{2} \theta}{\sigma_{v}^{2} M \beta_{j}\left|f_{j}\right|^{2}+\sigma_{n}^{2}}\right),
$$

which results in the expression given in (14). Following similar steps, the mean $\mu_{T_{\mathrm{KP}} \mid \mathcal{H}_{0}}$ under the null hypothesis in (13) can also be determined. The variance $\sigma_{T_{\mathrm{KP}}}^{2}$ is given by $\sigma_{T_{\mathrm{KP}}}^{2}=\mathbb{E}\left\{T_{\mathrm{KP}}^{2}(\tilde{\mathbf{y}}) \mid \mathcal{H}_{0}\right\}-\left(\mathbb{E}\left\{T_{\mathrm{KP}}(\tilde{\mathbf{y}}) \mid \mathcal{H}_{0}\right\}\right)^{2}$, where the quantity $\mathbb{E}\left\{T_{\mathrm{KP}}^{2}(\tilde{\mathbf{y}}) \mid \mathcal{H}_{0}\right\}$ in the above expression can be determined as

$$
\begin{aligned}
& \mathbb{E}\left\{T_{\mathrm{KP}}^{2}(\tilde{\mathbf{y}}) \mid \mathcal{H}_{0}\right\}=\mathbb{E}\left\{\left[\sum_{j=1}^{K} \Re\left(\frac{M a_{j}^{*} f_{j}^{*} \tilde{y}_{j}}{\sigma_{v}^{2} M \beta_{j}\left|f_{j}\right|^{2}+\sigma_{n}^{2}}\right)\right]^{2} \mid \mathcal{H}_{0}\right\} \\
& =\mathbb{E}\left\{\sum_{j=1}^{K}\left[\Re\left(\frac{M a_{j}^{*} f_{j}^{*}\left(\beta_{j} f_{j} v_{j}+\frac{1}{M} \mathbf{g}_{j}^{H} \mathbf{n}\right)}{M \sigma_{v}^{2} \beta_{j}\left|f_{j}\right|^{2}+\sigma_{n}^{2}}\right)\right]^{2}\right\} \\
& =\sum_{j=1}^{K} \frac{1}{2}\left(\frac{M \beta_{j}\left|a_{j}\right|^{2}\left|f_{j}\right|^{2}}{M \sigma_{v}^{2} \beta_{j}\left|f_{j}\right|^{2}+\sigma_{n}^{2}}\right)
\end{aligned}
$$

Substituting (63) and the value of $\mu_{T_{\mathrm{KP}} \mid \mathcal{H}_{0}}$ in $\sigma_{T_{\mathrm{KP}}}^{2}$, one can obtain the final expression of the variance $\sigma_{T_{\mathrm{KP}}}^{2}$ in (15).

\section{APPENDIX B \\ Proof of THEOREM 1}

The optimization problem in (18) can be efficiently solved using the Karush-Kuhn-Tucker (KKT) framework [42]. The corresponding Lagrangian can be formulated as

$$
L\left(p_{j}, \lambda, \mu_{j}\right)=\sum_{j=1}^{K} \frac{-M \beta_{j}\left|a_{j}\right|^{2} p_{j}}{M \beta_{j} p_{j} \sigma_{v}^{2}+\sigma_{n}^{2}}+\lambda\left(\sum_{j=1}^{K} p_{j}-P\right)+\sum_{j=1}^{K} \mu_{j} p_{j},
$$

and the KKT conditions can be expressed as

$$
\begin{aligned}
\frac{-M \beta_{j}\left|a_{j}\right|^{2} \sigma_{n}^{2}}{\left(M \beta_{j} p_{j} \sigma_{v}^{2}+\sigma_{n}^{2}\right)^{2}}+\lambda-\mu_{j} & =0, \\
\lambda\left(\sum_{j=1}^{K} p_{j}-P\right) & =0, \\
\sum_{j=1}^{K} p_{j}-P & =0, \\
\mu_{j} p_{j} & =0, \\
\mu_{j}, p_{j}, \lambda & \geq 0 .
\end{aligned}
$$




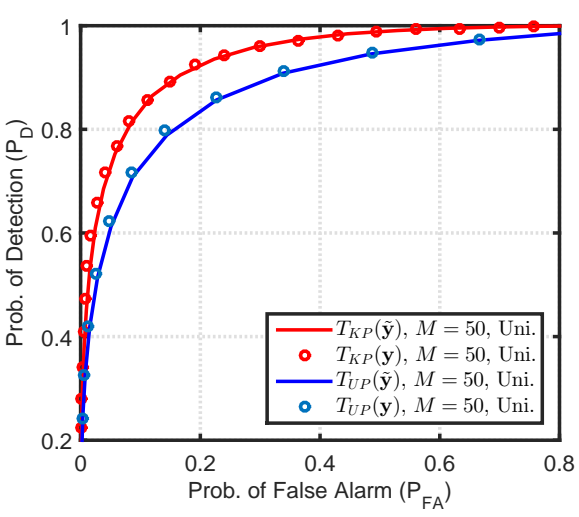

(a)

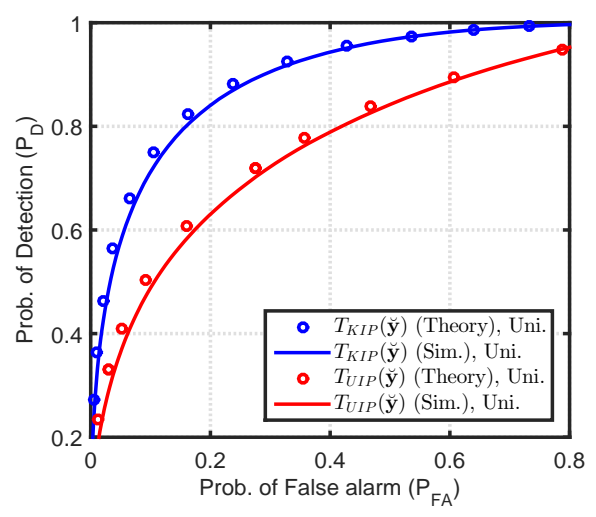

(b)

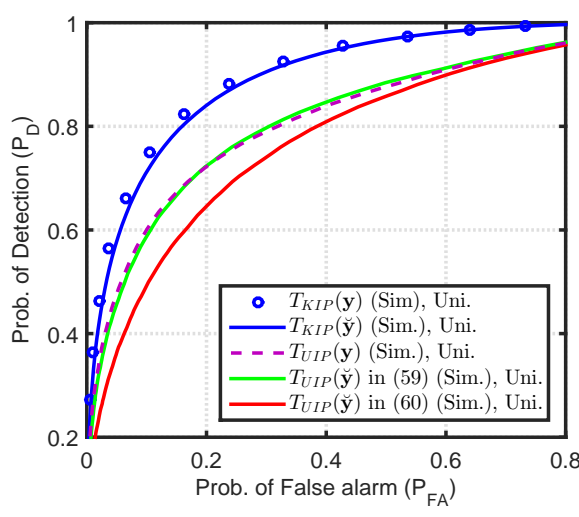

(c)

Fig. 6. ROC plot for comparing (a) detector $T_{\mathrm{KP}}(\tilde{\mathbf{y}})$ with the detector $T_{\mathrm{KP}}(\mathbf{y})$ and the detector $T_{\mathrm{UP}}(\tilde{\mathbf{y}})$ with the detector $T_{\mathrm{UP}}(\mathbf{y})$ using $M=50$ for $K=12$, $\sigma_{v}^{2}=0.2, \sigma_{n}^{2}=0.7$ and $P=1$. (b) detectors $T_{\mathrm{KIP}}(\breve{\mathbf{y}})$ and $T_{\mathrm{UIP}}(\breve{\mathbf{y}})$ in (45) and (60), respectively, for imperfect CSI with their analytical counterparts for a WSN with $K=12, M=50, \sigma_{v}^{2}=0.2, \sigma_{n}^{2}=0.7$ and $P=1$. (c) detectors $T_{\mathrm{KIP}}(\breve{\mathbf{y}})$ in (45) with the detector $T_{\mathrm{KIP}}(\mathbf{y})$ and the detector $T_{\mathrm{UIP}}(\breve{\mathbf{y}})$ in $(59)$ with the detector in (60) and the detector $T_{\mathrm{UIP}}(\mathbf{y})$, for a WSN with $K=12, M=50, \sigma_{v}^{2}=0.2, \sigma_{n}^{2}=0.7$ and $P=1$.

Using the above KKT conditions followed by straightforward manipulations yields the closed-form solution in (19).

\section{APPENDIX C}

\section{PROOF OF THEOREM 2}

$T_{\mathrm{UP}}^{\prime}(\tilde{\mathbf{y}})$ in (23) is Gaussian distributed under both the hypotheses, which can be described as

$$
\begin{aligned}
T_{\mathrm{UP}}^{\prime}(\tilde{\mathbf{y}}) & =\sum_{j=1}^{K} \Re\left(\frac{M f_{j}^{*} a_{j}^{*} \tilde{y}_{j}}{\sigma_{v}^{2} M \beta_{j}\left|f_{j}\right|^{2}+\sigma_{n}^{2}}\right) \\
& \sim\left\{\begin{array}{lll}
\mathcal{N}\left(\mu_{T_{\mathrm{UP}}^{\prime} \mid \mathcal{H}_{0}}, \sigma_{T_{\mathrm{UP}}^{\prime}}^{2}\right) & \text { under } & \mathcal{H}_{0} \\
\mathcal{N}\left(\mu_{T_{\mathrm{UP}}^{\prime} \mid \mathcal{H}_{1}}, \sigma_{T_{\mathrm{UP}}^{\prime}}^{2}\right) & \text { under } & \mathcal{H}_{1}
\end{array}\right.
\end{aligned}
$$

where the quantities $\mu_{T_{\mathrm{UP}}^{\prime} \mid \mathcal{H}_{0}}, \mu_{T_{\mathrm{UP}}^{\prime} \mid \mathcal{H}_{1}}$ and $\sigma_{T_{\mathrm{UP}}^{\prime}}^{2}$ in (25), (26) and (27), respectively, are obtained using the same procedure as described in Appendix A. Using the statistics in (69), the probability of detection $P_{D}$ can be formulated as

$$
\begin{aligned}
P_{D} & =\operatorname{Pr}\left\{\left|T_{\mathrm{UP}}^{\prime}(\tilde{\mathbf{y}})\right|>\gamma^{\prime \prime} \mid \mathcal{H}_{1}\right\} \\
& =\operatorname{Pr}\left\{T_{\mathrm{UP}}^{\prime}(\tilde{\mathbf{y}})>\gamma^{\prime \prime} \mid \mathcal{H}_{1}\right\}+\operatorname{Pr}\left\{T_{\mathrm{UP}}^{\prime}(\tilde{\mathbf{y}})<-\gamma^{\prime \prime} \mid \mathcal{H}_{1}\right\} \\
& =Q\left(\frac{\gamma^{\prime \prime}-\mu_{T_{\mathrm{UP}}^{\prime} \mid \mathcal{H}_{1}}}{\sigma_{T_{\mathrm{UP}}}}\right)+1-Q\left(\frac{-\gamma^{\prime \prime}-\mu_{T_{\mathrm{UP}}^{\prime} \mid \mathcal{H}_{1}}}{\sigma_{T_{\mathrm{UP}}^{\prime}}}\right) .
\end{aligned}
$$

Using the relation $Q(-x)=1-Q(x)$, (70) can be further simplified to obtain the final expression of $P_{D}$ in (24). The closed-form expression of the probability of false alarm $P_{F A}$ in (24) can be derived along similar lines.

\section{APPENDIX D}

\section{PROOF OF THEOREM 3}

Under both the hypotheses, the test statistic $T_{\mathrm{KP}}(\tilde{\mathbf{y}})$ in (11) for the detection of a known parameter, is distributed as $\mathcal{N}\left(\mu_{T_{\mathrm{KP}} \mid \mathcal{H}_{i}}, \sigma_{T_{\mathrm{KP}}}^{2}\right)$, where $i=0,1$. The equivalent test statistic obtained after appropriate scaling, i.e., $\bar{T}_{\mathrm{KP}}(\tilde{\mathbf{y}}) \triangleq \frac{T_{\mathrm{KP}}(\tilde{\mathbf{y}})}{\sigma_{T_{\mathrm{KP}}}}$, also follows the Gaussian distribution under both the hypotheses, which can be expressed as $\bar{T}_{\mathrm{KP}}(\tilde{\mathbf{y}}), \mathcal{H}_{i} \sim \mathcal{N}\left(\bar{\mu}_{T_{\mathrm{KP}} \mid \mathcal{H}_{i}}^{u}, 1\right)$. Furthermore, the scaled mean $\bar{\mu}_{T_{\mathrm{KP}} \mid \mathcal{H}_{i}}^{u}$ can be defined as $\bar{\mu}_{T_{\mathrm{KP}} \mid \mathcal{H}_{i}}^{u} \triangleq \frac{\mu_{T_{\mathrm{KP}} \mid \mathcal{H}_{i}}}{\sigma_{T_{\mathrm{KP}}}}$. Substituting the quantities $\mu_{T_{\mathrm{KP}} \mid \mathcal{H}_{1}}$ and
$\sigma_{T_{\mathrm{KP}}}$, given in (14) and (15), respectively, and using $p_{u}=$ $\left|f_{j}\right|^{2}$, the scaled mean $\bar{\mu}_{T_{\mathrm{KP}} \mid \mathcal{H}_{1}}^{u}$ for the alternative hypothesis $\mathcal{H}_{1}$ can be simplified as

$$
\bar{\mu}_{T_{\mathrm{KP}} \mid \mathcal{H}_{1}}=\sqrt{\sum_{j=1}^{K} \frac{2 p_{u} M \beta_{j}\left|a_{j}\right|^{2} \theta^{2}}{\sigma_{v}^{2} p_{u} M \beta_{j}+\sigma_{n}^{2}}} .
$$

Using the above expression, the probability of detection $\bar{P}_{D}$ corresponding to the detector $\bar{T}_{\mathrm{KP}}(\tilde{\mathbf{y}})$ can be expressed as $\bar{P}_{D}=Q\left(\gamma^{\prime}-\bar{\mu}_{T_{\mathrm{KP}} \mid \mathcal{H}_{1}}^{u}\right)$. The asymptotic probability of detection $P_{D}^{u}$ in the large antenna regime, given by (30), can be determined by evaluating the normalized mean. This can be simplified for hypothesis $\mathcal{H}_{1}$ by taking the limit as $M \rightarrow \infty$ of $\bar{\mu}_{T_{\mathrm{KP}} \mid \mathcal{H}_{1}}^{u}$, along with the scaling $p_{u}=\frac{\tilde{p}_{u}}{M}$, and can be expressed as

$$
\begin{aligned}
\tilde{\mu}_{T_{\mathrm{KP}} \mid \mathcal{H}_{1}}^{u} & =\left.\lim _{M \rightarrow \infty} \bar{\mu}_{T_{\mathrm{KP}} \mid \mathcal{H}_{1}}^{u}\right|_{p_{u}=\frac{\tilde{p}_{u}}{M}}=\left.\lim _{M \rightarrow \infty} \frac{\mu_{T_{\mathrm{KP}} \mid \mathcal{H}_{1}}}{\sigma_{T_{\mathrm{KP}}}}\right|_{p_{u}=\frac{\tilde{p}_{u}}{M}} \\
& =\lim _{M \rightarrow \infty} \sqrt{\sum_{j=1}^{K} \frac{2 \frac{\tilde{p}_{u}}{M} M \beta_{j}\left|a_{j}\right|^{2} \theta^{2}}{\sigma_{v}^{2}}},
\end{aligned}
$$

which on further simplification reduces to (31). The asymptotic probability of false alarm $P_{F A}^{u}$ in (30) can be derived along similar lines.

\section{APPENDIX E \\ PROOF OF THEOREM 6}

The $k$-th cumulant of $T_{\mathrm{KIP}}(\breve{\mathbf{y}})$ for hypothesis $\mathcal{H}_{i}$, where $i=0,1$, is given by

$$
\kappa_{k}^{i}=2^{k-1}(k-1) ! \sum_{j=1}^{K}\left(2\left(\frac{\lambda_{j, i}}{2}\right)^{k}+k\left(\frac{\lambda_{j, i}}{2}\right)^{k} \xi_{j, i}\right) .
$$

The mean, standard deviation, skewness and kurtosis of the test statistic $T_{\mathrm{KIP}}(\breve{\mathbf{y}})$ under both the hypotheses can be evaluated using the expression (71). Using (47), (48) and (71), the probability of detection $P_{D}$ can be determined as shown below.

$$
P_{D}=\operatorname{Pr}\left(\frac{\sum_{j=1}^{K} \frac{\lambda_{j, 1}}{2} \chi_{2}^{\prime 2}\left(\xi_{j, 1}\right)-\mu_{\chi^{\prime}}}{\sigma_{\chi^{\prime}}}>\frac{\tilde{\gamma}-\mu_{\chi^{\prime}}}{\sigma_{\chi^{\prime}}}\right)
$$




$$
\approx \operatorname{Pr}\left(\frac{\chi_{l_{\mathrm{KD}}}^{\prime 2}\left(\xi_{\mathrm{KD}}\right)-\mu_{\mathrm{KD} \mid \mathcal{H}_{1}}}{\sigma_{\mathrm{KD} \mid \mathcal{H}_{1}}}>\frac{\tilde{\gamma}-\mu_{\mathrm{KD} \mid \mathcal{H}_{1}}}{\sigma_{\mathrm{KD} \mid \mathcal{H}_{1}}}\right)
$$

where $\mu_{\chi^{\prime}}, \sigma_{\chi^{\prime}}$ denote the mean and standard deviation of the weighted sum of $K$ chi-square distributed random variables $\sum_{j=1}^{K} \frac{\lambda_{j, 1}}{2} \chi_{2}^{\prime 2}\left(\xi_{j, 1}\right)$. The above expression (72), for large number of antennas at the FC, can be further simplified to obtain the expression in (51). The quantities $\mu_{\mathrm{KD} \mid \mathcal{H}_{1}}$ and $\sigma_{\mathrm{KD} \mid \mathcal{H}_{1}}$ represent the mean and standard deviation of the chi-square random variable $\chi_{l_{\mathrm{KD}}}^{\prime 2}\left(\delta_{\mathrm{KD}}\right)$ and are defined in (53) and (55), respectively. The expression for $P_{F A}$ in (51) can be derived on similar lines.

\section{APPENDIX F \\ PROOF OF THEOREM 7}

For the detector in (60), the probability of detection $P_{D}$ is given by

$$
P_{D}=\operatorname{Pr}\left(\breve{\mathbf{y}}^{H} \mathbf{X} \breve{\mathbf{y}}>\tilde{\gamma}^{\prime} \mid \mathcal{H}_{1}\right) .
$$

To evaluate $P_{D}$, the above expression can be equivalently written as $P_{D}=\operatorname{Pr}\left(\breve{\mathbf{y}}^{\prime H} \mathbf{Z} \breve{\mathbf{y}}^{\prime}>\tilde{\gamma}^{\prime}\right)$, where the vector $\breve{\mathbf{y}}^{\prime}$ and the matrix $\mathbf{Z}$ are defined as $\breve{\mathbf{y}}^{\prime}=\mathbf{C}_{\boldsymbol{\eta}}^{-\frac{1}{2}} \breve{\mathbf{y}}$ and $\mathbf{Z}=$ $\mathbf{C}_{\boldsymbol{\eta}}^{\frac{1}{2}} \mathbf{X} \mathbf{C}_{\boldsymbol{\eta}}^{\frac{1}{2}}$, respectively. Under hypothesis $\mathcal{H}_{1}, \breve{\mathbf{y}}$ is distributed as $\breve{\mathbf{y}} \sim \mathcal{C N}\left(\mathbf{D}_{\hat{\mathrm{g}}} \mathbf{F a} \theta, \mathbf{C}_{\boldsymbol{\eta}}\right)$. Hence, $\breve{\mathbf{y}}^{\prime}$ also follows the complex Gaussian distribution, i.e., $\breve{\mathbf{y}}^{\prime} \sim \mathcal{C N}\left(\mathbf{C}_{\boldsymbol{\eta}}^{-\frac{1}{2}} \mathbf{D}_{\hat{\mathrm{g}}} \mathbf{F a} \theta, \mathbf{I}_{K}\right)$. Using the eigenvalue decomposition (EVD), the matrix $\mathbf{Z}$ can be defined as $\mathbf{Z}=\mathbf{U} \boldsymbol{\Sigma} \mathbf{U}^{H}$. On substituting the EVD of $\mathbf{Z}$, the expression of $P_{D}$ reduces to $P_{D}=\operatorname{Pr}\left(\breve{\mathbf{y}}^{\prime H} \mathbf{U} \boldsymbol{\Sigma} \mathbf{U}^{H} \breve{\mathbf{y}}^{\prime}>\right.$ $\left.\tilde{\gamma}^{\prime}\right)=\operatorname{Pr}\left(\breve{\mathbf{y}}_{u}^{H} \boldsymbol{\Sigma} \breve{\mathbf{y}}_{u}>\tilde{\gamma}^{\prime}\right)$, where the vector $\breve{\mathbf{y}}_{u}$ is defined as $\breve{\mathbf{y}}_{u}=\mathbf{U}^{H} \breve{\mathbf{y}}^{\prime}$. The transformed vector $\breve{\mathbf{y}}_{u}$ is distributed as $\breve{\mathbf{y}}_{u} \sim \mathcal{C N}\left(\mathbf{U}^{H} \mathbf{C}_{\boldsymbol{\eta}}^{-\frac{1}{2}} \mathbf{D}_{\hat{\mathbf{g}}} \mathbf{F a} \theta, \mathbf{I}_{K}\right)$. Hence, the expression of $P_{D}$ further simplifies to $P_{D}=\operatorname{Pr}\left(\sum_{j=1}^{K} \sigma_{j} \chi_{2}^{\prime 2}\left(\xi_{j}\right)>\tilde{\gamma}^{\prime}\right)$, where $\sigma_{j}$ is the $j$-th element of the eigenvalue matrix $\boldsymbol{\Sigma}$ and $\chi_{2}^{\prime 2}\left(\xi_{j}\right)$ is the non-central chi-squared random variable with two degrees of freedom and non-centrality parameter as $\xi_{j}$. Following the procedure in Appendix E, the probability of detection $P_{D}$ can be determined as $P_{D} \approx \operatorname{Pr}\left(\chi_{l_{\mathrm{UD}}}^{\prime 2}\left(\xi_{\mathrm{UD}}\right)>\right.$ $\left.\tilde{\gamma}^{\prime}\right)=Q_{\chi_{l_{\mathrm{UD}}^{\prime}}^{\prime 2}\left(\xi_{\mathrm{UD}}\right)}\left(\tilde{\gamma}^{\prime}\right)$, which is given in (61). The expression for the $P_{F A}$ in (61) can be derived similarly.

\section{REFERENCES}

[1] R. V. Kulkarni and G. K. Venayagamoorthy, "Particle swarm optimization in wireless-sensor networks: A brief survey," IEEE Trans. Systems, Man, and Cybernetics, Part C (Applications and Reviews), vol. 41, no. 2, pp. 262-267, Mar. 2011.

[2] M. Raza, N. Aslam, H. Le-Minh, S. Hussain, Y. Cao, and N. M. Khan, "A critical analysis of research potential, challenges, and future directives in industrial wireless sensor networks," IEEE Commun. Surveys Tutorials, vol. 20, no. 1, pp. 39-95, 2018.

[3] W. Li and H. Dai, "Distributed detection in wireless sensor networks using a multiple access channel," IEEE Trans. Signal Process., vol. 55, no. 3, pp. 822-833, Mar. 2007.

[4] J. F. Chamberland and V. V. Veeravalli, "Decentralized detection in sensor networks," IEEE Trans. Signal Process., vol. 51, no. 2, pp. 407416, Feb. 2003.

[5] J. F. Chamberland and V. V. Veeravalli, "Asymptotic results for decentralized detection in power constrained wireless sensor networks," IEEE J.Sel. Areas Commun., vol. 22, no. 6, pp. 1007-1015, Aug. 2004.

[6] R. Niu, B. Chen, and P. K. Varshney, "Fusion of decisions transmitted over Rayleigh fading channels in wireless sensor networks," IEEE Trans. Signal Process., vol. 54, no. 3, pp. 1018-1027, Mar. 2006.
[7] M. Gastpar, "Uncoded transmission is exactly optimal for a simple Gaussian "sensor" network," IEEE Trans. Inf. Theory, vol. 54, no. 11, pp. 5247-5251, Nov. 2008.

[8] Z. Quan, W. Ma, S. Cui, and A. H. Sayed, "Optimal linear fusion for distributed detection via semidefinite programming," IEEE Trans. Signal Process., vol. 58, no. 4, pp. 2431-2436, Apr. 2010.

[9] W. Li and H. Dai, "Distributed detection in wireless sensor networks using a multiple access channel," IEEE Trans. Signal Process., vol. 55, no. 3, pp. 822-833, Mar. 2007.

[10] C. Tepedelenlioglu and S. Dasarathan, "Distributed detection over Gaussian multiple access channels with constant modulus signaling," IEEE Trans. Signal Process., vol. 59, no. 6, pp. 2875-2886, Jun. 2011.

[11] M. K. Banavar, A. D. Smith, C. Tepedelenliolu, and A. Spanias, "Distributed detection over fading MACs with multiple antennas at the fusion center," in 2010 IEEE Int. Conf. Acoust., Speech, Signal Process., Mar. 2010, pp. 2894-2897.

[12] M. K. Banavar, A. D. Smith, C. Tepedelenlioglu, and A. Spanias, "On the effectiveness of multiple antennas in distributed detection over fading MACs," IEEE Trans. Wireless Commun., vol. 11, no. 5, pp. 1744-1752, May 2012.

[13] I. Nevat, G. W. Peters, and I. B. Collings, "Distributed detection in sensor networks over fading channels with multiple antennas at the fusion centre," IEEE Trans. Signal Process., vol. 62, no. 3, pp. 671683, Feb. 2014.

[14] D. Ciuonzo, G. Romano, and P. S. Rossi, "Channel-aware decision fusion in distributed MIMO wireless sensor networks: Decode-and-fuse vs. decode-then-fuse," IEEE Trans. Wireless Commun., vol. 11, no. 8, pp. 2976-2985, Aug. 2012.

[15] M. A. Al-Jarrah, M. A. Yaseen, A. Al-Dweik, O. A. Dobre, and E. Alsusa, "Decision fusion for IoT-based wireless sensor networks," IEEE Internet of Things Journal, vol. 7, no. 2, pp. 1313-1326, 2020.

[16] A. Patel, H. Ram, A. K. Jagannatham, and P. K. Varshney, "Robust cooperative spectrum sensing for MIMO cognitive radio networks under CSI uncertainty," IEEE Trans. Signal Process., vol. 66, no. 1, pp. 18-33, Jan. 2018

[17] Q. Cheng, B. Chen, and P. K. Varshney, "Detection performance limits for distributed sensor networks in the presence of nonideal channels," IEEE Trans. Wireless Commun., vol. 5, no. 11, pp. 3034-3038, Nov. 2006.

[18] A. R. Reibman and L. W. Nolte, "Optimal detection and performance of distributed sensor ," IEEE Trans. Aerosp. Electron. Syst., vol. AES-23, no. 1, pp. 24-30, Jan. 1987

[19] J. N. Tsitsiklis, "Decentralized detection," Advances in Signal Processing, , vol. 2, H. V. Poor and J. B. Thomas, editors, JAI Press, pp. 297-344, 1993.

[20] R. Viswanathan and P. K. Varshney, "Distributed detection with multiple sensors part I. Fundamentals," Proceedings of the IEEE, vol. 85, no. 1, pp. 54-63, Jan. 1997.

[21] J. Fang, H. Li, Z. Chen, and S. Li, "Optimal precoding design and power allocation for decentralized detection of deterministic signals," IEEE Trans. Signal Process., vol. 60, no. 6, pp. 3149-3163, Jun. 2012.

[22] D. Gesbert, M. Kountouris, R. W. H. Jr., C. b. Chae, and T. Salzer, "Shifting the MIMO paradigm," IEEE Signal Process. Mag., vol. 24, no. 5 , pp. 36-46, Sep. 2007.

[23] F. Rusek, D. Persson, B. K. Lau, E. G. Larsson, T. L. Marzetta, O. Edfors, and F. Tufvesson, "Scaling up MIMO: Opportunities and challenges with very large arrays," IEEE Signal Process. Mag., vol. 30, no. 1, pp. 40-60, Jan. 2013.

[24] H. Q. Ngo, E. G. Larsson, and T. L. Marzetta, "Energy and spectral efficiency of very large multiuser MIMO systems," IEEE Trans. Commun., vol. 61, no. 4, pp. 1436-1449, Apr. 2013.

[25] L. Lu, G. Y. Li, A. L. Swindlehurst, A. Ashikhmin, and R. Zhang, "An overview of massive MIMO: Benefits and challenges," IEEE J. of Sel. Topics Signal Process., vol. 8, no. 5, pp. 742-758, Oct. 2014.

[26] T. L. Marzetta, "Noncooperative cellular wireless with unlimited numbers of base station antennas," IEEE Trans. Wireless Commun., vol. 9 no. 11 , pp. 3590-3600, Nov. 2010.

[27] F. Jiang, J. Chen, A. L. Swindlehurst, and J. A. López-Salcedo, "Massive MIMO for wireless sensing with a coherent multiple access channel," IEEE Trans. Signal Process., vol. 63, no. 12, pp. 3005-3017, Jun. 2015.

[28] A. Shirazinia, S. Dey, D. Ciuonzo, and P. S. Rossi, "Massive MIMO for decentralized estimation of a correlated source," IEEE Trans. Signal Process., vol. 64, no. 10, pp. 2499-2512, May 2016.

[29] G. Ding, X. Gao, Z. Xue, Y. Wu, and Q. Shi, "Massive MIMO for distributed detection with transceiver impairments," IEEE Trans. Veh. Technol., vol. 67, no. 1, pp. 604-617, Jan. 2018. 
[30] D. Ciuonzo, P. S. Rossi, and S. Dey, "Massive MIMO channel-aware decision fusion," IEEE Trans. Signal Process., vol. 63, no. 3, pp. 604 619, Feb. 2015.

[31] D. Ciuonzo, A. Aubry, and V. Carotenuto, "Rician MIMO channel and jamming-aware decision fusion," IEEE Trans. Signal Process., vol. 65, no. 15, pp. 3866-3880, Aug. 2017.

[32] A. Chawla, A. Patel, A. K. Jagannatham, and P. K. Varshney, "Robust distributed detection in massive MIMO wireless sensor networks under CSI uncertainty," in IEEE 88th Veh. Technol. Conf., Aug. 2018, pp. 1-5.

[33] A. Chawla, A. Patel, A. K. Jagannatham, and P. K. Varshney, "Distributed detection in massive MIMO wireless sensor networks under perfect and imperfect CSI," IEEE Trans. Signal Process., vol. 56, no. 9, pp. 4124-4140, Jun. 2019.

[34] I. Dey, D. Ciuonzo, and P. S. Rossi, "Wideband collaborative spectrum sensing using massive MIMO decision fusion," IEEE Trans. Wireless Commun., pp. 1-1, 2020.

[35] J. Xiao, S. Cui, Z. Luo, and A. J. Goldsmith, "Linear coherent decentralized estimation," IEEE Transactions on Signal Processing, vol. 56, no. 2, pp. 757-770, 2008 .

[36] H. Q. Ngo, E. G. Larsson, and T. L. Marzetta, "Aspects of favorable propagation in massive MIMO," in 2014 22nd European Signal Process.
Conf. (EUSIPCO), Sep. 2014, pp. 76-80.

[37] S. M. Kay, Fundamentals of statistical signal processing, volume 2: Detection theory. New Jersey: Prentice-Hall Inc, 1993.

[38] A. Chawla, A. S. Sarode, A. K. Jagannatham, and L. Hanzo, "Technical Report: Distributed parameter detection in massive MIMO wireless sensor networks relying on imperfect CSI,” IIT Kanpur, Tech. Rep., 2020, [Online]. Available: http://www.iitk.ac.in/mwn/documents/TR_DDP_MMIMO_2020.pdf.

[39] B. Picinbono, "On deflection as a performance criterion in detection," IEEE Trans. Aerosp. Electron. Syst., vol. 31, no. 3, pp. 1072-1081, Jul. 1995.

[40] S. M. Kay, Fundamentals of statistical signal processing: Estimation theory. Upper Saddle River, NJ, USA: Prentice-Hall, Inc., 1993.

[41] H. Liu, Y. Tang, and H. H. Zhang, "A new chi-square approximation to the distribution of non-negative definite quadratic forms in non-central normal variables," Computational Statistics and Data Analysis, vol. 53, no. 4, pp. 853-856, Feb. 2009.

[42] S. Boyd and L. Vandenberghe, Convex optimization. New York, NY, USA: Cambridge University Press, 2004 